\title{
Neurons secrete miR-132-containing exosomes to regulate brain vascular integrity
}

\author{
Bing $\mathrm{Xu}^{1,{ }^{*}}$, Yu Zhang ${ }^{1,2,{ }^{*}}$, Xu-Fei $\mathrm{Du}^{1}$, Jia $\mathrm{Li}^{1}$, Hua-Xing $\mathrm{Zi}^{1,2}$, Ji-Wen Bu ${ }^{1}$, Yong Yan ${ }^{1,3}$, Hua Han ${ }^{4}$, Jiu-Lin Du ${ }^{1,2,3}$ \\ ${ }^{1}$ Institute of Neuroscience, State Key Laboratory of Neuroscience, Center for Excellence in Brain Science and Intelligence Tech- \\ nology, Chinese Academy of Sciences, 320 Yue-Yang Road, Shanghai 200031, China; ${ }^{2}$ University of Chinese Academy of Sciences, \\ 19A Yu-Quan Road, Beijing 100049, China; ${ }^{3}$ School of Life Science and Technology, ShanghaiTech University, 319 Yue-Yang Road, \\ Shanghai 200031, China; ${ }^{4}$ Institute of Automation, Center for Excellence in Brain Science and Intelligence Technology, Chinese \\ Academy of Sciences, 95 Zhong-guan-cun East Road, Beijing 100190, China
}

Vascular integrity helps maintain brain microenvironment homeostasis, which is critical for the normal development and function of the central nervous system. It is known that neural cells can regulate brain vascular integrity. However, due to the high complexity of neurovascular interactions involved, understanding of the neural regulation of brain vascular integrity is still rudimentary. Using intact zebrafish larvae and cultured rodent brain cells, we find that neurons transfer miR-132, a highly conserved and neuron-enriched microRNA, via secreting exosomes to endothelial cells (ECs) to maintain brain vascular integrity. Following translocation to ECs through exosome internalization, miR-132 regulates the expression of vascular endothelial cadherin (VE-cadherin), an important adherens junction protein, by directly targeting eukaryotic elongation factor 2 kinase (eef $2 \mathrm{k}$ ). Disruption of neuronal miR-132 expression or exosome secretion, or overexpression of vascular eef $2 k$ impairs VE-cadherin expression and brain vascular integrity. Our study indicates that miR-132 acts as an intercellular signal mediating neural regulation of the brain vascular integrity and suggests that the neuronal exosome is a novel avenue for neurovascular communication.

Keywords: brain vascular integrity; neuron; endothelial cell; exosome; miR-132; eef2k; zebrafish

Cell Research (2017) 27:882-897. doi: 10.1038/cr.2017.62; published online 21 April 2017

\section{Introduction}

Brain vascular endothelial cells (ECs) connect with each other through junction proteins and are enveloped by surrounding pericytes and astrocytes to form the blood-brain barrier (BBB) [1-4]. The BBB restricts material exchange between the brain and blood vessels to maintain the homeostasis of the brain microenvironment, which is crucial for the normal development and function of the central nervous system (CNS) [1-4]. It is generally believed that the development of brain vasculature, including its integrity, is tightly regulated by surrounding neural tissues. In the past several years, several signaling pathways, in particular the Wnt and Sonic Hedgehog

\footnotetext{
*These two authors contributed equally to this work.

Correspondence: Jiu-lin Du

E-mail: forestdu@ion.ac.cn

Received 29 November 2016; revised 25 February 2017; accepted 26

March 2017; published online 21 April 2017
}

(Shh) pathways, were found to be implicated in this neural regulation $[4,5]$. During embryogenesis, neural progenitors secrete Wnt ligands, which activate canonical Wnt signaling in ECs and contribute to the maturation of brain vasculature [6-8]. At postnatal stages, astrocyte-secreted Shh further promotes vascular integrity of the brain by activating Hedgehog signaling in ECs through the receptor Patched-1 [9]. However, the understanding of the neural regulation of brain vascular integrity is still rudimentary.

Exosomes are small membrane vesicles that originate from the inward budding of endosomal membranes [10]. Diverse types of cells including neurons can secrete exosomes, which transfer functional proteins [11, 12], mRNAs and/or microRNAs (miRNAs) $[13,14]$ to recipient cells. Exosome-mediated material transfer has been recognized as an important route for intercellular communication [15]. Recent studies show that neurons can secrete exosomes to regulate neural development $[16$, 17]. For example, motor neuron terminals in Drosophila 
larvae release exosomes to promote the maturation of neuromuscular junctions [11, 18]. As blood vessels in the developing brain are sparsely covered by mural cells, it is possible that neuronal exosomes can be directly taken up by adjacent ECs to mediate the neural regulation of brain vascular development.

In the present study, we found that, neurons transfer $m i R-132$, an evolutionarily conserved and neuron-enriched miRNA [19, 20], into ECs via secreting exosomes to regulate the brain vascular integrity. Knockdown or mutation of miR-132 caused severe intracranial hemorrhage and disruption of brain vascular integrity in zebrafish larvae with reduced expression of the adherens junction protein vascular endothelial cadherin (VE-cadherin, also known as Cdh5) and its intracellular partner $\beta$-catenin. The defect in the brain vascular integrity was mimicked by neuron-specific reduction of $m i R-132$. Interestingly, up- and downregulation of the $m i R-132$ level in neurons resulted in a coordinated change of the $m i R-132$ level in ECs, and EC-specific reduction of $m i R-132$ also impaired Cdh5 expression as well as the brain vascular integrity. Combining experiments on cultured rodent brain cells, we found that neurons secreted $m i R$-132-containing exosomes, which were then internalized into brain ECs, leading to an increased level of $m i R-132$ in ECs, and impairment of exosome secretion in zebrafish larvae caused intracranial hemorrhage. Furthermore, we identified eukaryotic elongation factor 2 kinase (eef $2 k$ ) as a direct target of $m i R-132$. Gain- and loss-of-function experiments showed that eef $2 k$ in ECs mediated the action of miR-132 on Cdh5 expression and brain vascular integrity. This study discovers a previously unidentified function of $m i R-132$ and reveals that neuronal exosomes serve as a novel carrier in mediating the neural regulation of brain vascular integrity.

\section{Results}

\section{MiR-132 is necessary for the brain vascular integrity in} larval zebrafish

MiRNAs are small non-coding RNAs that post-transcriptionally regulate the expression of target mRNAs [21]. MiR-132 is a neuron-enriched miRNA and plays crucial roles in neural development and plasticity [19, 20]. MiR-132 is evolutionarily conserved among animal species and highly expressed in the brain of zebrafish larvae (Supplementary information, Figure S1A and S1B; see also ref. [22]).

To examine whether $m i R-132$ is important for brain vascular development, we downregulated the expression of miR-132 using two morpholino oligonucleotides (MOs), which targeted mature miR-132 (“miR-132 MO", see also ref. [23]) or precursor $m i R-132$ loop (" $m i R-132$ loop MO", Supplementary information, Figure S1C). The expression of $m i R-132$ in zebrafish larvae was efficiently downregulated by these MOs (Supplementary information, Figure S1D; $P<0.05$ ). In comparison with embryos injected with a control MO ("Ctrl MO"), a substantial proportion of $m i R-132$ morphants displayed intracranial hemorrhage (Figure $1 \mathrm{~A}$ and $1 \mathrm{C}$; Ctrl MO: $3.3 \% \pm 0.7 \% ; m i R-132$ MO: $38.0 \% \pm 2.8 \% ; m i R-132$ loop MO: $37.1 \% \pm 7.9 \%$; mean \pm SEM, $P<0.001)$ without any marked embryonic death or defects in gross morphology (Supplementary information, Figure S1E and S1F). To demonstrate the leakage of blood cells in the brain, we knocked down miR-132 expression in double transgenic zebrafish $\operatorname{Tg}$ (Flk1:eGFP;Gata1:DsRed) larvae, in which ECs express enhanced green fluorescent protein (eGFP) and blood cells express DsRed. This allowed us to observe the accumulation of DsRed-expressing blood cells in brain ventricles (Figure 1B). Blood cell leakage in brain ventricles was further confirmed by transmission electron microscopy (TEM) (Supplementary information, Figure S1G).

Next, we investigated whether the intracranial hemorrhage induced by $m i R-132$ knockdown was due to the impairment of brain vascular integrity. To this end, we first injected a solution containing DAPI and Alex568 dextran (10 kDa molecular mass) into the blood circulation system through the common cardinal vein in $\mathrm{Tg}(\mathrm{Flk} 1$ :eGFP) larvae 3 days post fertilization (dpf) and evaluated the degree of vascular leakage by counting DAPI-positive nuclei of brain parenchymal cells 30 min later [24]. A significant increase of DAPI-positive parenchymal cell nuclei as well as dextran leakage was observed in miR-132 morphants (Figure 1D, 1E and Supplementary information, Figure $\mathrm{S} 1 \mathrm{H} ; P<0.001)$. Second, we injected red fluorescent beads $(100 \mathrm{~nm}$ in diameter) into the blood circulation system of $\mathrm{Tg}(\mathrm{Flk} 1$ :eGFP) larvae and immediately monitored bead leakage by in vivo time-lapse confocal imaging. In contrast to control embryos, red fluorescent beads were readily observed to leak out from the brain vessels of miR-132 morphants (Supplementary information, Figure S1I and S1J). More importantly, 17 out of 18 leakage events observed occurred at functional vessels which already exhibited blood flow (Figure 1F and Supplementary information, Figure S1J; data obtained from $9 \mathrm{miR}-132$ morphants). This suggested that the vascular leakage in brains of miR-132 morphants is due to defects in the integrity of brain vasculature. Third, we examined the ultra-structure of brain vessels using TEM. EC-EC contacts in control larvae showed numerous and extensive junctions (Figure $1 \mathrm{G}$ and $1 \mathrm{H}$ ) whereas junctions between brain ECs 
A

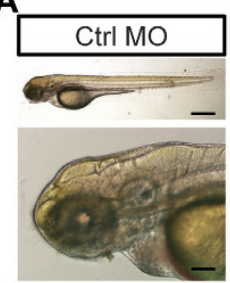

B

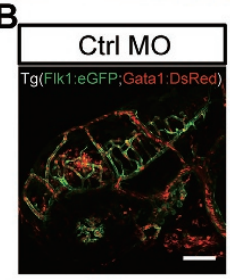

$\mathbf{F}$

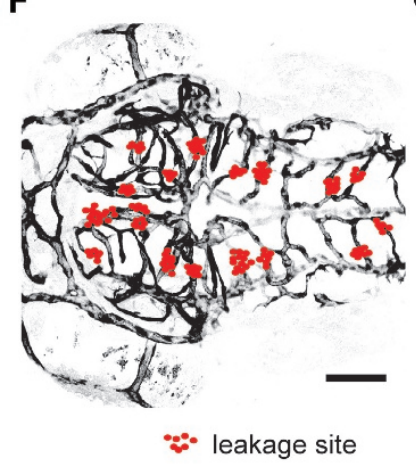

C
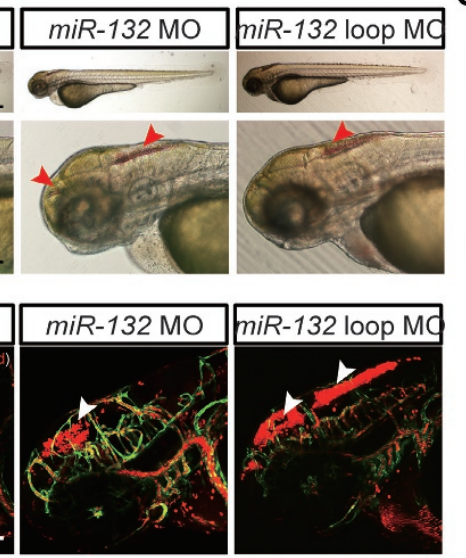

G

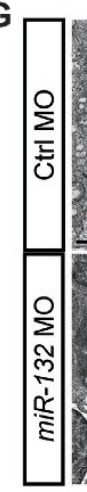

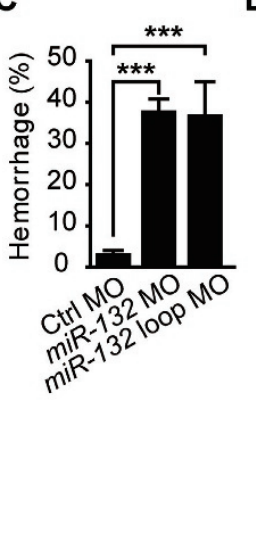

D

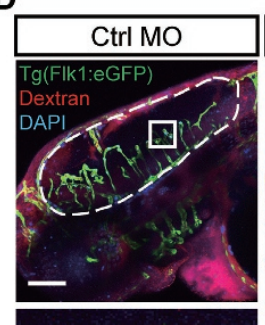

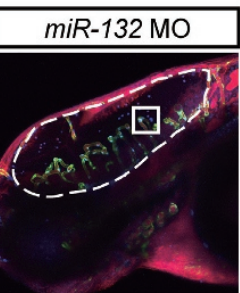

$E$

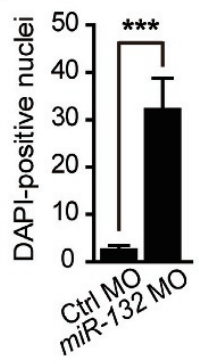

Figure 1 MiR-132 knockdown and mutation impair brain vascular integrity of larval zebrafish. (A, B) Representative images showing that miR-132 knockdown with morpholino oligonucleotides (MOs) caused intracranial hemorrhage (arrowheads) in wildtype larvae (A) and blood cell leakage (arrowheads) in the brain of Tg(Flk1:eGFP;Gata1:DsRed) larvae (B). The images were taken from larvae at 3 days post fertilization (dpf). (C) Summary of the intracranial hemorrhage effect of miR-132 knockdown. The experiments were repeated 4-8 times, and at each time $>89$ embryos were examined for each group. (D, E) Representative images and summary showing that miR-132 knockdown caused more DAPI-positive brain parenchymal nuclei (blue) in 3-dpf Tg(Flk1:eGFP) larvae, in which a mixture of DAPI and Alex-568 dextran were injected into the blood circulation system. In $\mathbf{D}$, the area outlined by the rectangles in the top are enlarged in the bottom, and the number of DAPI-positive nuclei in the area outlined by the dashed lines were counted. 13 control embryos and 18 morphants were analyzed in E. (F) Spatial distribution of leakage sites (red dots) in all 9 miR-132 morphants examined by in vivo time-lapse confocal imaging. (G-I) Transmission electron microscopy (TEM) images showing the ultrastructural changes of brain vessels in miR132 morphants. The areas outlined by the red dashed rectangles in G were enlarged in (H, "Ctrl MO") and (I, "miR-132 MO"). The green arrowheads in $\mathbf{G}$ point to the abnormal sites of the vessel luminal side, and the red arrowheads in I point to the abnormal junctions between vascular endothelial cells (ECs). (J) Rescue effect of miR-132 RNA overexpression on intracranial hemorrhage defects in miR-132 morphants. The experiments were repeated four times, and at each time $>75$ embryos were examined for each group. (K) Summary of the intracranial hemorrhage effect of miR-132 mutations generated by co-injection of zebrafish codon-optimized Cas9 mRNA and miR-132 gRNA. The experiments were repeated 3-5 times, and for each time $>21$ embryos were examined for each group. Scale bar, $400 \mu \mathrm{m}$ (top) and $100 \mu \mathrm{m}$ (bottom) (A), $100 \mu \mathrm{m}$ (B), $100 \mu \mathrm{m}$ (top) and $10 \mu \mathrm{m}$ (bottom) (D), $100 \mu \mathrm{m}$ (F), $1 \mu \mathrm{m}$ (G), $200 \mathrm{~nm}$ (H and I). Error bars, SEM. n.s., no significant; ${ }^{* * *} P<0.001$ (one-way ANOVA with post hoc Bonferroni's multiple comparison test for $\mathbf{C}$ and $\mathbf{K}$; unpaired two-tailed Student's $t$-test for $\mathbf{E}$; one-way ANOVA with post hoc Tukey's multiple comparison test for $\mathbf{J}$ ).

in hemorrhagic miR-132 morphants was discontinuous, junction density was reduced, and the cleft between ECs was increased (Figure $1 \mathrm{G}$ and 1I). The luminal side of brain vessels in miR-132 morphants was tortuous and rough (Figure 1G). Consistent with a previous finding that $m i R-132$ can facilitate pathological angiogenesis [25], we also found the brain vascular density was decreased in $m i R-132$ morphants (Supplementary information, Figure $\mathrm{S} 2 ; P<0.01)$.

Because only about $40 \%$ of $m i R-132$ morphants ex- 
hibited severe intracranial hemorrhage (Figure 1C), we examined the level of $m i R-132$ in hemorrhagic and non-hemorrhagic miR-132 morphants, and found that the expression of $m i R-132$ was comparable in the two groups (Supplementary information, Figure S3A). We noted, however, in those miR-132 morphants that did not exhibit hemorrhage, brain vascular integrity was also disrupted as evidenced by a significant increase of DAPI leakage from the brain vasculature (Supplementary information, Figure S3B and S3C; $P<0.01)$.

This defect of brain vascular integrity was a specific consequence of downregulation of $m i R-132$, because co-injection of miR-132 RNA with miR-132 MO alleviated the hemorrhagic phenotype in morphants (Figure $1 \mathrm{~J} ; P<0.001)$. Moreover, miR-132 mutations induced by co-injecting miR-132 guide RNA (gRNA) and zebrafish codon-optimized $\operatorname{Cas} 9$ mRNA also led to a significant increase of intracranial hemorrhage in F0 embryos (Figure $1 \mathrm{~K}$ and Supplementary information, Figure S4; $P<$ $0.001)$. Taken together, these results indicate that $m i R$ 132 is necessary for the integrity of the brain vasculature.

Cdh5 and its intracellular partner $\beta$-catenin are involved in brain vascular integrity

The maintenance of brain vascular integrity requires tight connections between ECs through junction proteins [2]. In hemorrhagic miR-132 morphants, the expression of Cdh5, an important component of adherens junctions [26], but not Claudin-5, Occludin, ZO-1 or N-cadherin was markedly reduced (Figure $2 \mathrm{~A}-2 \mathrm{C} ; P<0.01$ ). To further confirm changes in adherens junction proteins, we examined the expression of $\alpha$-catenin and $\beta$-catenin, which are important intracellular partners of Cdh5 [26]. The expression of $\beta$-catenin was reduced in $m i R-132$ morphants $(P<0.05)$, whereas we observed no significant change for $\alpha$-catenin (Figure 2A and 2B). Similar to the effects of miR-132 knockdown, Cdh5 morphants also displayed increased intracranial hemorrhage (see also ref. [27]) and DAPI leakage (Figure 2D, 2E and Supplementary information, Figure $\mathrm{S} 5 ; P<0.001$ ).

We then chose to examine the effect of $m i R-132$ knockdown on endothelial transcytosis and pericytes that can also regulate vascular permeability in the brain [4]. Zebrafish vessel-specific 1 (vsgl) is an ortholog of the mammalian plasmalemma vesicle associated protein (Plvap) [28], which is a critical mediator of endothelial transcytosis $[29,30]$. We found that vsgl mRNA was reduced rather than increased in miR-132 morphants (Figure 2F; $P<0.01$ ), suggesting that at least transcytosis is not enhanced by miR-132 knockdown. To examine changes in coverage of brain vessels by pericyte, we first generated pdgfr $\beta$ :Gal4 knockin zebrafish, named Ki(p- dgfr $\beta: G a 14)$, in which Gal4 is specifically expressed in pericytes (Materials and Methods). By crossing Ki(pdgfrß:Gal4) with $\operatorname{Tg}(\mathrm{UAS}: \mathrm{GFP}$;Fli1:DsRed), we generated larvae in which pericytes express GFP and ECs express DsRed. These allowed us to determine that pericyte coverage was not significantly changed in $m i R-132$ morphants (Figure 2G and $2 \mathrm{H}$ ). Collectively, these results suggest that $m i R-132$ regulates the brain vascular integrity through affecting adherens junction proteins rather than transcytosis or pericytes.

Neuronal miR-132 regulates brain vascular integrity via affecting vascular miR-132 level

In mammals, $m i R-132$ is enriched in neurons $[19,20]$. Combining flow cytometry and real-time PCR analysis, we found that, in zebrafish larvae, expression of $m i R-132$ was much higher in neurons than in ECs (Figure 3A; $P$ $<0.001$ ). To study the consequences of downregulating the level of $m i R-132$ in neurons, we transiently expressed $m i R-132$ sponge (miR-132-S) in neurons. The miR-132-S was inserted into the $3^{\prime}$ untranslated region ( $3^{\prime}$ UTR) of the reporter gene tdTomato $(t d T)$ and driven by the promoter of the neuron-specific gene $H u C$ ("HuC:tdT-miR132-S"; Materials and Methods). The sponge contained ten repeats of the miR-132 antisense sequence and efficiently inhibited $m i R-132$ function by chelating $m i R-132$ (Supplementary information, Figure S6; $P<0.001$ ) [31]. In comparison with control larvae, in which neurons expressed $t d T$ (“'HuC:tdT"), larvae with neurons expressing $m i R-132-S$ exhibited severe intracranial hemorrhage (Figure 3B and 3C; $P<0.01$ ) and increased DAPI leakage (Figure 3D and 3E; $P<0.05$ ) and reduced Cdh5 expression (Figure 3F; $P<0.05$ ). Thus, neuronal $m i R-132$ is necessary for the integrity of brain vasculature. Interestingly, reduction of $m i R-132$ in neurons by transiently expressing $H u C: t d T-m i R-132-S$ also decreased the level of $m i R-132$ in ECs (Figure 3G; $P<0.05$ ). Conversely, overexpression of $m i R-132$ in neurons, through the transient expression of $H u C: t d T-m i R-132$, increased the $m i R$ 132 level in ECs (Figure 3G; $P<0.05$ ). The non-overlapping expression pattern of $H u C: t d T$ and Flk1:eGFP ensured that the coordinated changes in endothelial $m i R$ 132 were not due to the non-specific expression of $\mathrm{HuC}$ plasmids in ECs (Supplementary information, Figure S7). Considering that less than $5 \%$ neurons in the brain were labeled by $H u C$ promoter-driven transient gene expression, the effects of neuronal manipulations on $m i R$ 132 level in ECs are underestimated.

As ECs are the main constituent of the brain vasculature, we thus proposed that miR-132 in neurons may regulate the brain vascular integrity by affecting $m i R$ 132 levels in ECs. We then examined whether miR-132 
A

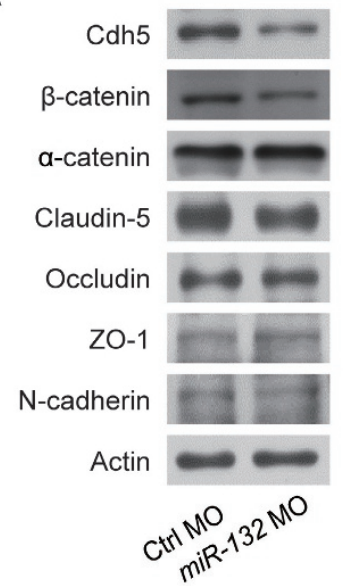

B

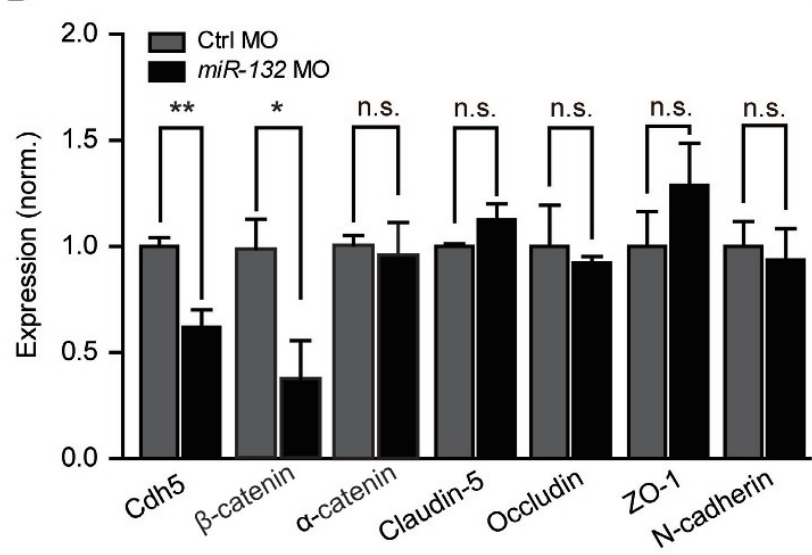

C

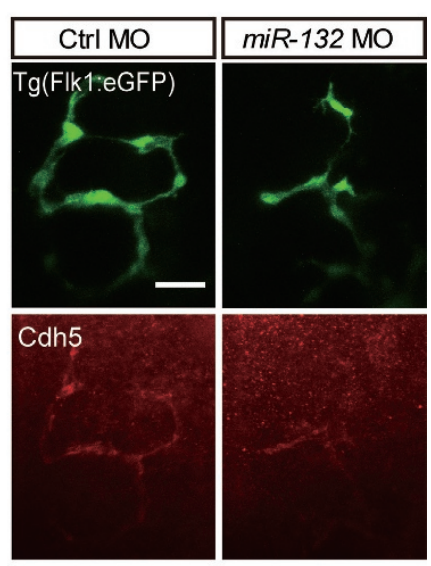

D

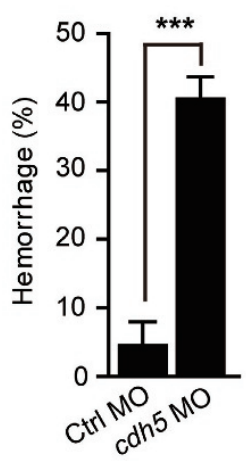

E

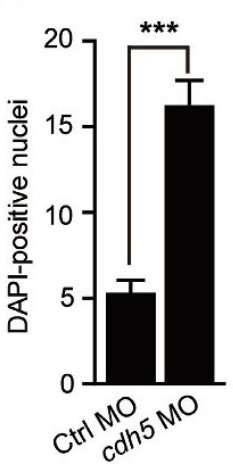

$\mathbf{F}$

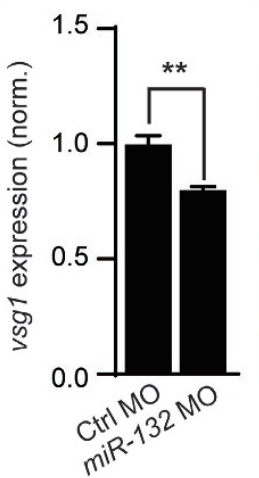

G

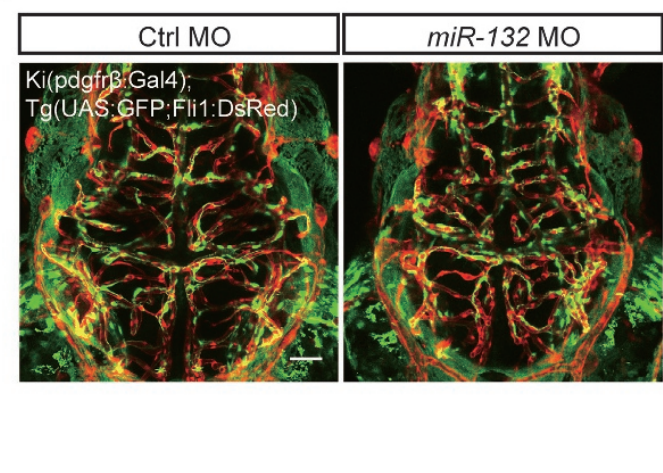

$\mathrm{H}$

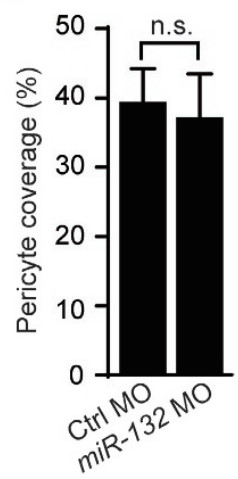

Figure 2 Involvement of Cdh5 in miR-132 knockdown-induced defects of brain vascular integrity. (A-C) Effects of miR-132 knockdown on the expression of junction proteins. Representative blots (A) and summary (B) of Western blot analyses. The experiments were repeated 3-8 times. (C) Cdh5 immunostaining in 3-dpf Tg(Flk1:eGFP) larvae. (D and E) Effects of Cdh5 knockdown on intracranial hemorrhage (D) and DAPI leakage (E) in 3-dpf larvae. The experiments were repeated 6 times and at each time $>49$ embryos were examined for each group in D. 23 control embryos and 24 morphants were analyzed in E. (F) Effect of miR-132 knockdown on vsg1 expression. The experiments were repeated three times. (G, H) Representative projected confocal images $(\mathbf{G})$ and summary $(\mathbf{H})$ showing no significant effect of miR-132 knockdown on the pericyte coverage of brain blood vessels. 3-dpf larvae generated by crossing Ki(pdgfr $\beta$ :Gal4) with Tg(UAS:GFP;Fli1:DsRed) were used, and GFP and DsRed are expressed in pericytes and ECs, respectively. 6 control embryos and 6 miR-132 morphants were analyzed. Scale bar, $25 \mu \mathrm{m}$ (C), $50 \mu \mathrm{m}$ (G). Error bars, SEM. n.s., not significant; ${ }^{\star} P<0.05,{ }^{* \star} P<0.01$, ${ }^{* \star} P<0.001$ (unpaired two-tailed Student's $t$-test for B, D, E, F and H).

in ECs can also regulate the brain vascular integrity. To address this point, we specifically downregulated the level of $m i R-132$ in ECs by transiently expressing $m i R$ 132-S driven by the promoter of the EC-specific gene Flk1 ("Flk1:tdT-miR-132-S"). In comparison with $t d T$ expression in ECs ("Flk1:tdT"), miR-132-S expression in ECs resulted in severe intracranial hemorrhage (Figure $3 \mathrm{H} ; P<0.01$ ) and reduction of $\mathrm{Cdh} 5$ expression (Figure 3I; $P<0.01)$. This indicates that $m i R-132$ in ECs can regulate expression of $\mathrm{Cdh} 5$ and the integrity of the brain vasculature. Together, these results suggest that neuronal miR-132 may regulate brain vascular integrity through coordinately affecting $m i R-132$ level in ECs.

\section{Exosomes transfer neuronal miR-132 from neurons to ECs}

Exosomes are newly identified vehicles for intercellular communication [15]. It was reported that neurons can transfer functional materials to recipient cells via secreting exosomes $[11,32]$ leading us to postulate that neuronal exosomes may transfer miR-132 from neurons to ECs. To test this hypothesis, we isolated exosomes from the conditioned medium of cultured primary rat cortical neurons (see Materials and Methods). TEM and nanopar- 


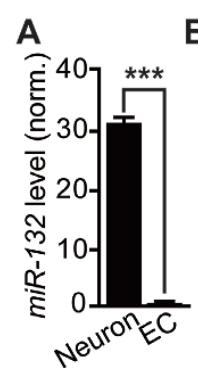

B
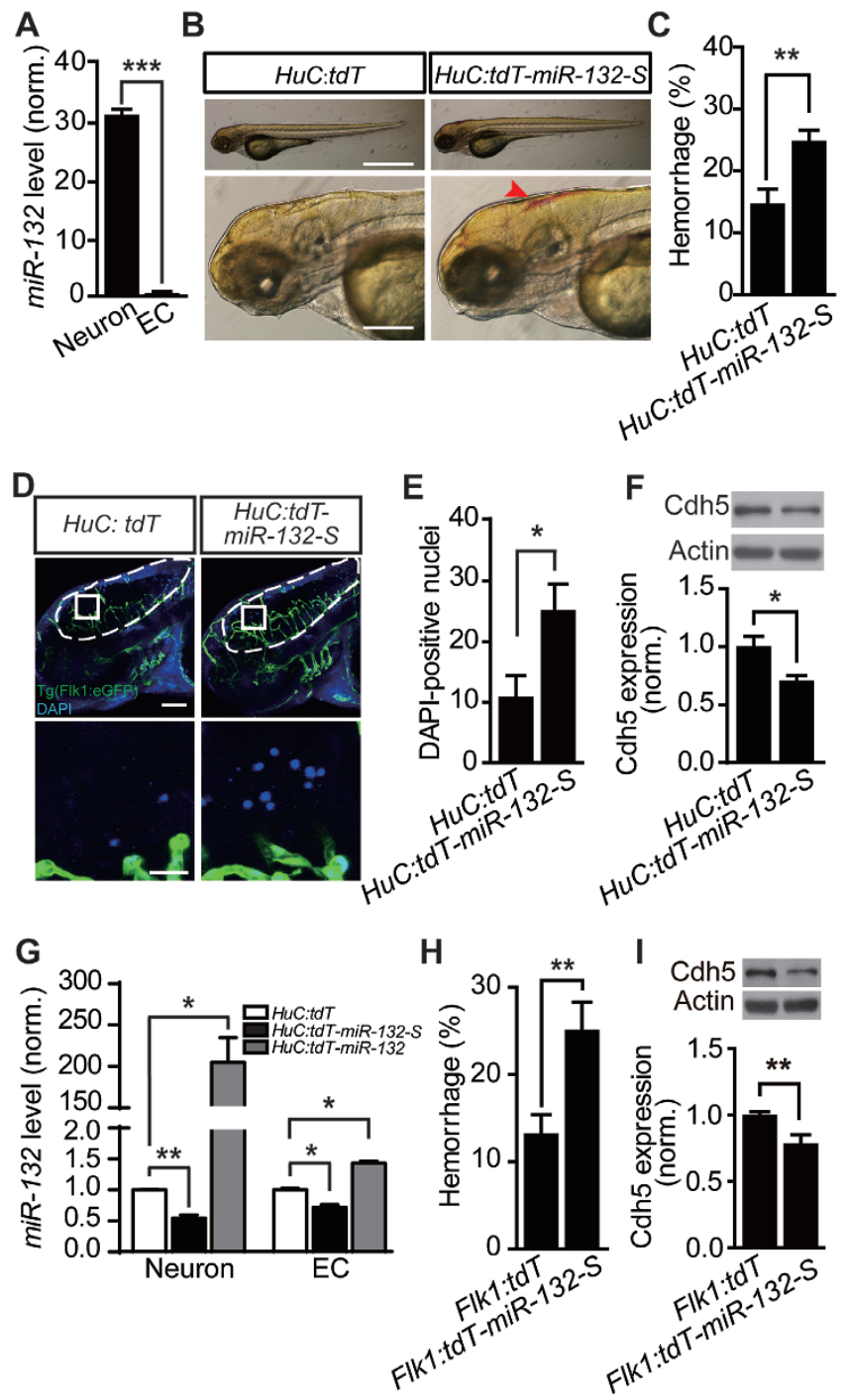

\section{I}

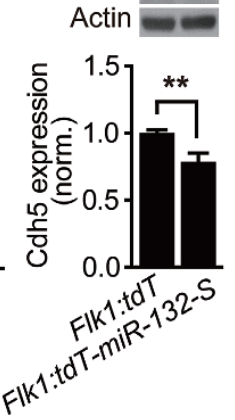

ticle tracing analysis of the purified pellets identified small membrane vesicles with an average diameter of $112 \pm 60 \mathrm{~nm}$ (mean $\pm \mathrm{SD}$; Figure 4A and 4B), suggesting the identity of exosomes. Furthermore, in comparison with cell lysates, vesicle preparations showed intensive expression of the exosomal markers Alix and CD63 but not the endoplasmic reticulum marker Calnexin (Figure 4C). Importantly, miR-132 was detected in the isolated neuronal exosome and its level was increased with the increased amount of exosomes (Figure 4D; $P<0.001$ ).

To address whether neuronal exosomes containing $m i R-132$ can be internalized into ECs, we first labeled purified exosomes with the green fluorescent lipid dye PKH67 and incubated them with the mouse brain microvascular EC line (b.End3). After incubation, green fluorescence-positive puncta were observed in both sparse and confluent cultured b.End 3 cells (Figure 4E
Figure 3 Neuronal miR-132 regulates brain vascular integrity by affecting endothelial miR-132 level. (A) Relative expression level of $m i R-132$ in zebrafish neurons and ECs, which were sorted by flow cytometry from 3-dpf $\mathrm{Tg}$ (HuC:GFP) or Tg(Flk1:eGFP) larvae, respectively. The experiments were repeated three times. (B-F) Effects of miR-132 sponge (miR-132-S)-mediated neuron-specific miR-132 downregulation on intracranial hemorrhage (B, C), DAPI leakage (D, E), and Cdh5 expression (F) in zebrafish larvae. The sponge was inserted into the tdTomato $(t d T)$ 3' UTR and driven by the HuC promoter ("HuC:tdT-miR132-S"). Expression of $t d T$ in neurons ("HuC:tdT") served as a control. The experiments were repeated 10 times in $\mathbf{C}$ and 5 times in $\mathbf{F}$, and 11 embryos injected with HuC:tdT and 8 embryos injected with HuC:tdT-miR-132-S were analyzed in E. (G) Coordinated changes of miR-132 level in zebrafish neurons and ECs, both of which were sorted from 3-dpf Tg(Flk1:eGFP) larvae transiently expressing HuC:tdT, HuC:tdT-miR-132-S or HuC:tdT-miR-132. Expression of HuC:tdT-miR-132-S or HuC:t$d T-m i R-132$ was used to down- or upregulate $m i R-132$ level in zebrafish neurons, respectively. The experiments were repeated 3-4 times. $(\mathbf{H}, \mathbf{I})$ Effects of miR-132-S expression in ECs on intracranial hemorrhage $(\mathbf{H})$ and Cdh5 expression $(\mathbf{I})$ in zebrafish larvae. The experiments were repeated nine times in $\mathbf{H}$ and six times in I. For each time, > 14 embryos were examined for each group in $\mathbf{H}$. Scale bar, $400 \mu \mathrm{m}$ (top) and $100 \mu \mathrm{m}$ (bottom) (B), $100 \mu \mathrm{m}$ (top) and $20 \mu \mathrm{m}$ (bottom) (D). Error bars, SEM. * $P<0.05$, ${ }^{* *} P<0.01,{ }^{* * *} P<0.001$ (unpaired two-tailed Student's $t$-test for A, C, E, F, H and I; one-way ANOVA with post hoc Bonferroni's multiple comparison test for $\mathbf{G}$ ).

and Supplementary information, Figure S8A). Exosome internalization was further confirmed by incubating b.End3 cells with exosomes purified from neurons in which CD63-GFP fusion protein was expressed. This lead to the appearance of GFP-expressing exosomes in b.End3 cells (Figure 4F). Next, by transfecting neurons with carboxyfluorescein (FAM)-tagged $m i R-132$, we observed fluorescence-positive exogenous $m i R-132$ in neuronal exosome-treated b.End3 cells (Figure 4G). Furthermore, incubation with neuronal exosomes significantly increased the level of $m i R-132$ in b.End 3 cells (Figure $4 \mathrm{H} ; P<0.05$ ). This increase was not affected by co-treatment with the RNA polymerase II inhibitor DRB (5, 6-dichloro-1-b-D-ribofuranosylbenzimidazole; Figure $4 \mathrm{H} ; P=0.9)$, suggesting neuronal exosomes can transfer mature $m i R-132$ to ECs. Using a luciferase assay, we found that neuronal exosomes repressed the luciferase activity of a miR-132 sensor in ECs, indicating that the transferred neuronal exosomal $m i R-132$ is functional in ECs (Supplementary information, Figure S8B; $P<0.05$ ). Consistently, we also found that the expression of Cdh5 in ECs was increased after treatment with neuronal exosomes (Figure 4I; $P<0.05$ ). Collectively, these results indicate that neuronal exosomes can transfer miR-132 into ECs. 
By overexpressing or downregulating miR-132 in cultured cortical neurons, we found that the miR-132 level in isolated neuronal exosomes positively correlated with that in neurons (Figure 4J and 4K; $P<0.01$ ). Moreover, the level of miR-132 in b.End3 cells could be further increased after incubation with the exosomes isolated from neurons overexpressing $m i R-132$ (Figure 4L; $P<0.001$ ). These in vitro findings show that neurons can regulate the level of $m i R-132$ in ECs through exosome-mediated intercellular transfer of $m i R-132$.

To demonstrate whether neuronal exosomes are involved in the regulation of brain vascular integrity in vivo, we labeled neuronal exosomes in zebrafish larvae via transiently expressing CD63-GFP driven by the $\mathrm{HuC}$ promoter ("HuC:CD63-GFP"). The neuron-specific distribution of GFP-positive puncta showed that CD63GFP can be used to trace neuronal exosomes (Supplementary information, Figure S8C). We then expressed $H u C: C D 63-G F P$ in $\mathrm{Tg}(\mathrm{Flk} 1: \mathrm{RFP})$ zebrafish larvae, in which vascular ECs expressed red fluorescent protein (RFP). In vivo time-lapse confocal imaging showed that GFP-positive neuronal exosomes approached brain vessels and ultimately entered ECs (Figure 5A). To further examine the role of exosomes in regulating brain vascular integrity, we reduced exosome release by targeting neutral sphingomyelinase 2 (nSMase2), which is required for the budding of exosomes into multi-vesicular bodies [33]. Treatment with the nSMase2 inhibitor spiroepoxide $(10 \mu \mathrm{M})$ or $n$ SMase 2 knockdown with MO led to severe intracranial hemorrhage accompanied with decreased expression of miR-132 in ECs of zebrafish larvae (Figure 5B, 5C and Supplementary information, Figure S8D$\mathrm{S} 8 \mathrm{~F} ; P<0.05)$. Taken together, these in vitro and in vivo findings suggest that neuronal exosomes containing $m i R$ 132 can mediate neuronal regulation of brain vascular integrity.

MiR-132 targets eef $2 k$ in ECs to regulate Cdh5 expression and brain vascular integrity

To search for the direct target of miR-132 that is involved in the regulation of brain vascular integrity, we identified 1253 upregulated genes in miR-132 morphants by performing microarray analysis (accession number for the microarray data: GSE85291 in NCBI) and predicted 848 target genes by using the Microcosm database. Among these predicted targets, 21 candidate genes (12 annotated) overlapped (Supplementary Information, Figure S9A). We then performed an in vitro luciferase assay for the 12 annotated genes. Consistent with the microarray data and the prediction that the zebrafish eef $2 k$ 3'UTR contains two miR-132 binding sites (Figure $6 \mathrm{~A}$ ), we found that $m i R-132$ markedly repressed the luciferase activity of the eef2k 3'UTR (Supplementary Information, Figure S9B). The eef $2 k$ encodes a calcium/ calmodulin-dependent protein kinase, which phosphorylates eukaryotic elongation factor 2 (eEF2) to inhibit translational elongation $[34,35]$. Both in vitro and in vivo reporter assays showed that eef $2 k 3^{\prime}$ UTR was sufficient to confer $m i R-132$ regulation (Figure 6B and Supplementary information, Figure S10A and S10B; $P<0.001$ ), and the second binding site was indispensable (Figure 6A and $6 \mathrm{C})$. Importantly, the expression of eEF2K in zebrafish larvae was decreased or increased by the overexpression or knockdown of $m i R-132$, respectively (Figure 6D and $6 \mathrm{E} ; P<0.05)$. These results indicate that eef $2 k$ is a direct target of $m i R-132$.

We then examined whether eEF2K mediates the effect of $m i R-132$ on the brain vascular integrity. Application of the eEF2K inhibitor NH125 $(2.5 \mu \mathrm{M})$ significantly alleviated the hemorrhagic defect in miR-132 morphants (Figure 6F; $P<0.001$ ). On the other hand, treatment with the eEF2K non-specific activator rapamycin $(2 \mu \mathrm{M})$ led to intracranial hemorrhage in wildtype larvae (Figure 6F; $P<0.05)$ and further aggravated the hemorrhagic defect of $m i R-132$ morphants (Figure 6F; $P<0.001$ ). Moreover, knockdown of eef $2 k$ alleviated the intracranial hemorrhage (Figure 6G; $P<0.01$ ), DAPI leakage (Figure 6H and Supplementary information, Figure S10C; $P<0.05$ ) and reduction of Cdh5 expression (Figure 6I; $P<0.05$ ) in $m i R-132$ morphants. More importantly, by combining flow cytometry and Western blotting, we found that the expression of eEF2K in ECs was significantly increased after sponge-mediated reduction of $m i R-132$ in neurons (Figure $6 \mathrm{~J} ; P<0.05$ ). Furthermore, EC-specific overexpression of Eef2k ("Flk1:tdT-P2A-Eef $2 k$ ") also caused intracranial hemorrhage (Figure $6 \mathrm{~K} ; P<0.01$ ), DAPI leakage (Figure 6L and Supplementary information, Figure S10D; $P<0.001$ ), and reduction of Cdh5 expression (Figure $6 \mathrm{M} ; P<0.01$ ). Taken together, these results indicate that eef $2 k$ is a direct target of $m i R-132$ in ECs to regulate the integrity of brain vasculature.

\section{Discussion}

Our study identifies a novel mechanism for the neural regulation of brain vascular integrity. Through secreting exosomes, neurons can translocate $m i R-132$ to ECs to regulate the expression of the vascular junction protein Cdh 5 by targeting eef $2 k$ (Figure 7). It highlights that neuronal exosomes can serve as an important mediator for neurovascular communication.

Novel function of miR-132 in regulating brain vascular integrity 
A
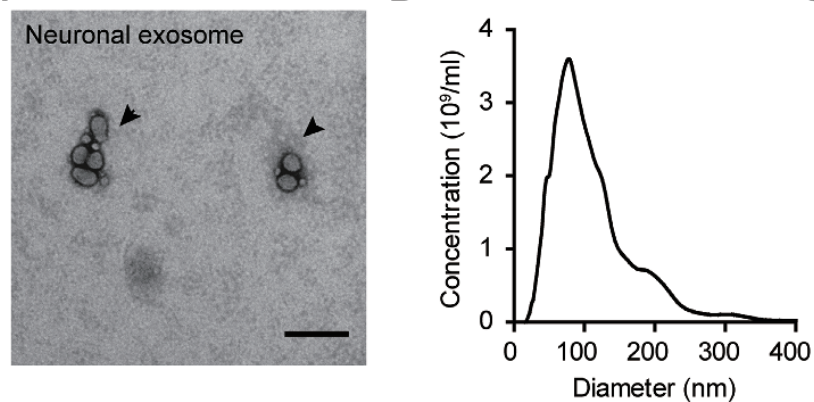

E

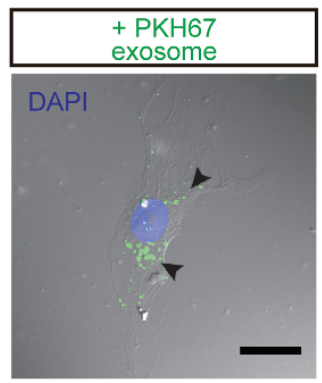

F

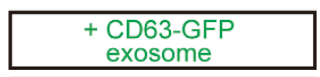

G
C

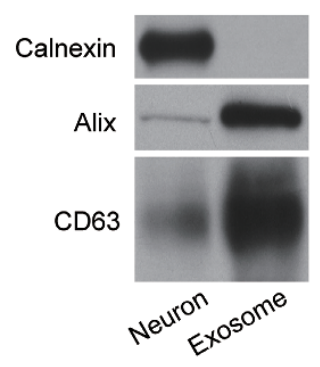

D

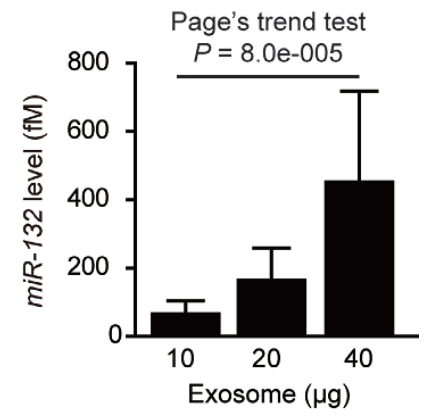

H

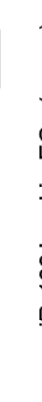

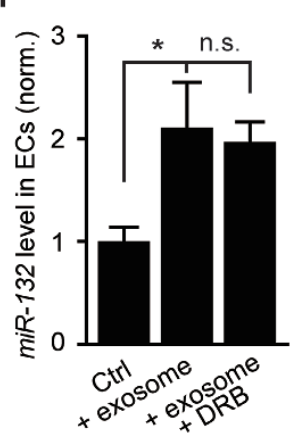

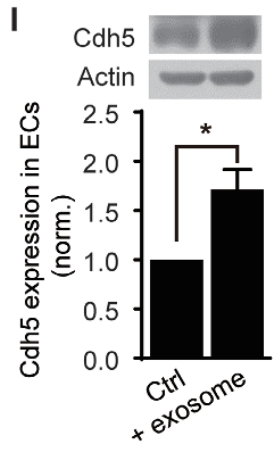

J

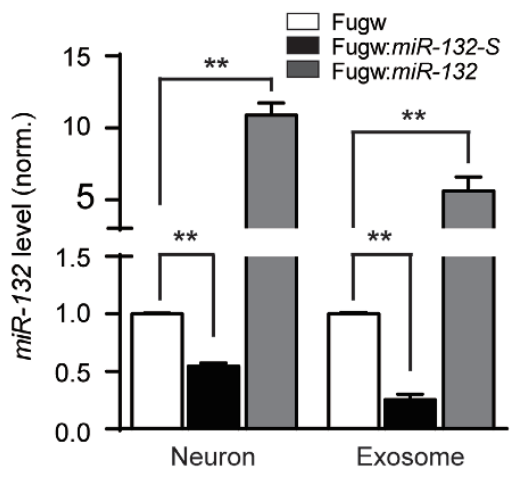

K

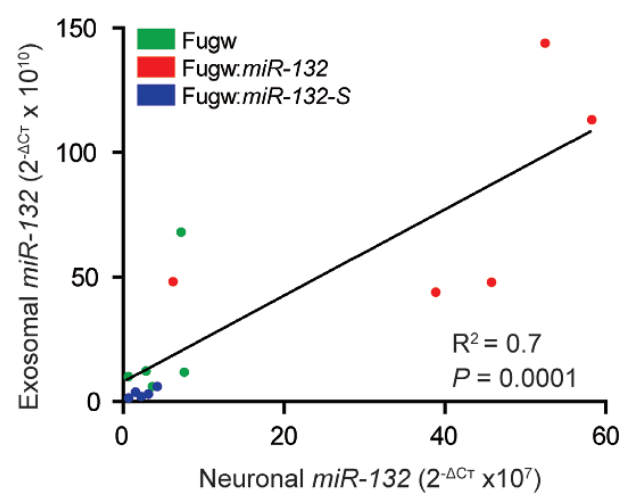

L

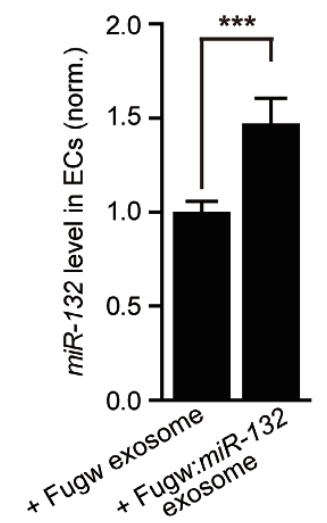

Figure 4 Neuronal exosomes transfer miR-132 from neurons to ECs. (A-C) Characterization of neuron-derived exosomes by transmission electron microscopy (TEM) (A), nanoparticle tracing analysis (B) and Western blotting with equal amounts of samples (C). The exosomes were isolated from the conditioned medium of cultured primary rat cortical neurons. Both Alix and CD63 are exosomal markers, and Calnexin is an endoplasmic reticulum marker. (D) MiR-132 level measured with different amount of neuronal exosomes. The experiments were repeated five times. (E, F) Internalization of PKH67 (E) or CD63GFP (F) labeled neuronal exosomes (arrowheads) into the mouse brain microvascular EC line (b.End3). DAPI staining (in blue) was performed to visualize the nuclei of b.End3 cells. (G) Existence of FAM-tagged miR-132 (arrowheads) in b.End3 cells, which were incubated with exosomes isolated from neurons transfected with FAM-tagged miR-132. (H) Relative level of miR-132 in b.End3 cells incubated with PBS ("Ctrl"), neuronal exosomes alone ("+ exosome") (200x), or neuronal exosomes plus the RNA polymerase II inhibitor DRB ("+ exosome + DRB") (200x). The experiments were repeated nine times. (I) Relative expression of Cdh5 in human umbilical vein endothelial cells (HUVECs) incubated with PBS ("Ctrl") and neuronal exosomes (“+ exosome”) (200x). The experiments were repeated four times. (J, K) Coordinated changes of miR-132 level in the cultured rat cortical neurons and their secreted exosomes. The line in $\mathbf{K}$ represents linear regression with $P=0.0001$. Cultured neurons were transfected with Fugw:miR-132-S or Fugw:miR-132 to, respectively, down- or up-regulate miR-132 levels, and transfection with Fugw served as a control. The experiments were repeated five times. (L) Relative level of miR132 in b.End3 cells incubated with exosomes isolated from neurons transfected with Fugw vector ("Fugw exosome") or Fugw:miR-132 ("Fugw:miR-132 exosome") (50×). The experiments were repeated three times. Scale bar, $100 \mathrm{~nm}$ (A), $25 \mu \mathrm{m}$ (E-G). Error bars, SEM. n.s., no significant; ${ }^{*} P<0.05,{ }^{* *} P<0.01,{ }^{* * *} P<0.001$ (Page's trend tests were applied for $\mathbf{D}$; oneway ANOVA with post hoc Tukey's multiple comparison test for $\mathbf{H}$ and $\mathbf{J}$; unpaired two-tailed Student's $t$-test for $\mathbf{I}$ and $\mathbf{L}$ ). 
A

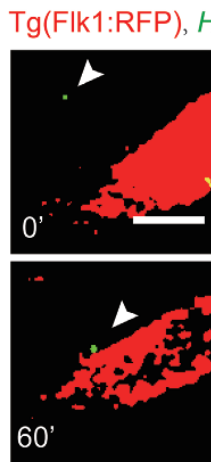

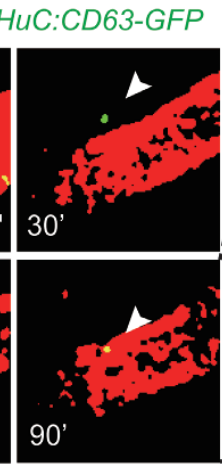
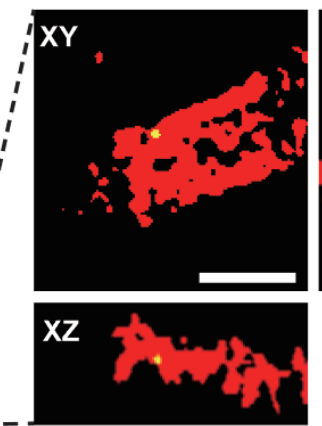

B

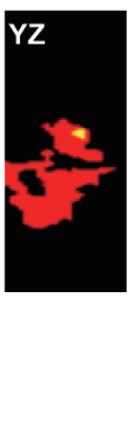

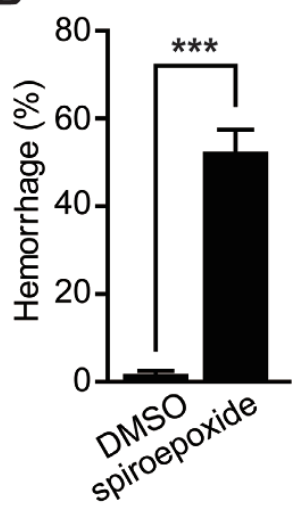

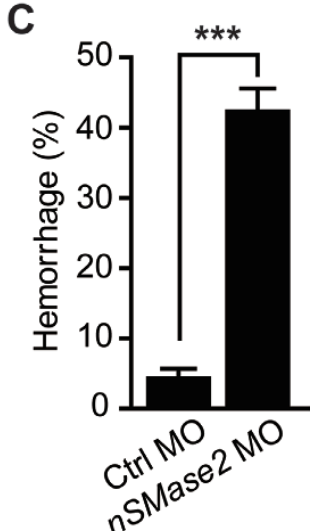

Figure 5 Neuronal exosomes are translocated to brain ECs in larval zebrafish. (A) In vivo time-lapse confocal images showing that a GFP-positive neuronal exosome (white arrowheads) approaches and enters RFP-positive ECs in the brain. The images were taken from a 3-dpf Tg(Flk1:RFP) zebrafish larva, in which HuC:CD63-GFP was transiently expressed to label neuronal exosomes. To trace the moving GFP-positive exosome at each time point, single-slice images at different optical sections are shown at the Left. To confirm the exosome internalization into ECs, three-dimensional views of the single-slice image at $90^{\prime}$ are shown at the Right. (B, C) Intracranial hemorrhage effects of nSMase2 blockade with spiroepoxide (B) or nSMase 2 knockdown with MO (C) in zebrafish. The nSMase2 is required for the budding of exosomes into multi-vesicular bodies. The experiments were repeated seven times in $\mathbf{B}$ and six times in $\mathbf{C}$. Scale bar, $5 \mu \mathrm{m}(\mathbf{A})$. Error bars, SEM. ${ }^{* * *} P<0.001$ (unpaired two-tailed Student's t-test for B and C).

MiR-132, a neuron-enriched miRNA, plays important roles in the development and activity-dependent plasticity of neural systems in a cell-autonomous manner. At a cellular level, $m i R-132$ regulates neurite growth and arborization [20, 36, 37], and synaptic structure and function $[19,38]$. At a system level, $m i R-132$ is essential for experience-dependent structural and functional plasticity of the visual cortex $[39,40]$. In the present study, we found that miR-132 exerts a non-cell-autonomous function in regulating the integrity of the brain vasculature. It can be transferred from neurons to brain ECs to promote the brain vascular integrity. As the impairment of brain vascular integrity will lead to the ingress of plasma components into the brain and compromise synaptic and neuronal functions $[1,2]$, the regulation of brain vascular integrity by $m i R-132$ may indirectly contribute to the role of $m i R-132$ in neural development and function.

The brain microenvironment is so complicated that different cell types and molecules are coordinated to regulate the development of brain vasculature. Besides neurons, pericytes and astrocytes are important for the development and maintenance of the integrity of brain vasculature [29, 30, 41]. However, there is no direct evidence for the existence of astrocytes in zebrafish larvae [42]. Here we found that knockdown of miR-132 did not affect the pericyte coverage, suggesting no direct involvement of pericytes in the regulation of brain vascular integrity by $m i R-132$. However, previous reports showed that $m i R-132$ was also expressed in pericytes and glial cells $[43,44]$. Thus, it is of interest to examine whether $m i R-132$ in pericytes and glial cells can also regulate brain vascular integrity.

\section{Emerging roles of neural systems in brain vascular in- tegrity}

During development, a few neural tissue-derived proteins were found to promote brain vascular development $[4,5]$. During embryogenesis, neural progenitor cells can secrete Wnt ligands to regulate the development of brain vasculature and BBB through activation of canonical Wnt signaling in ECs $[6,7]$. During postnatal development, astrocytes can release Shh to promote the brain vascular integrity by acting on EC Patched-1 receptors [9]. In addition, both pericytes and astrocytes can release Angiopoietin-1 that binds to endothelial Tie-2 receptors to improve brain vascular integrity $[45,46]$. Our study uncovers a new type of signal, i.e. neuron-derived miR$\mathrm{NA}$, which is involved in the neural regulation of brain vascular integrity. As the expression of $m i R-132$ is positively regulated by sensory experience and neural activity [38-40], it is of interest to examine whether enriched environment or enhanced neural activity can promote the development of brain vascular integrity by increasing $m i R-132$ levels. As miR-132 expression is kept at a high level in the brain [39], it is possible that the mechanism we have identified may also contribute to the mainte- 
A
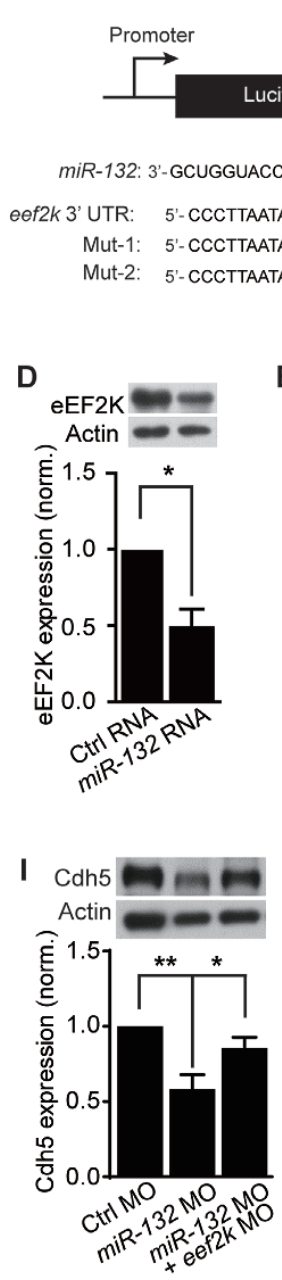

E

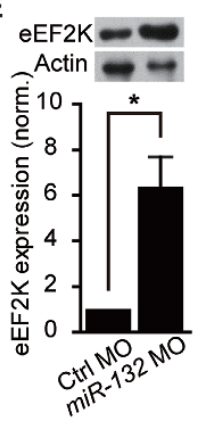

B

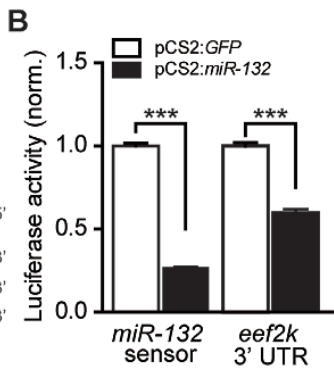

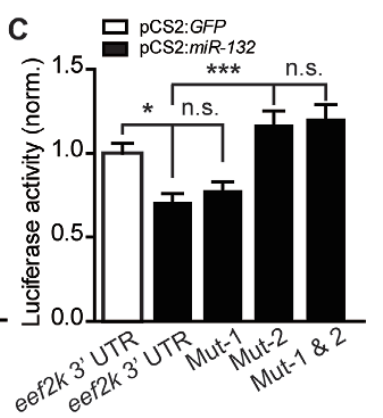

$\mathbf{F}$

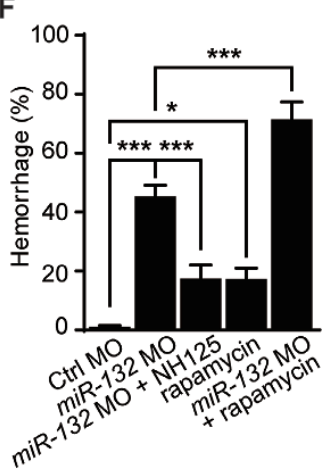

K

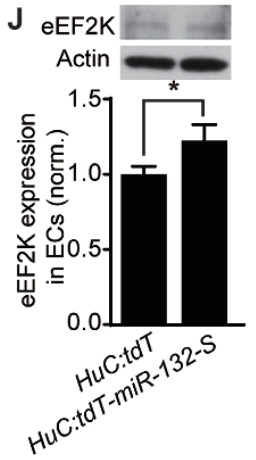

G

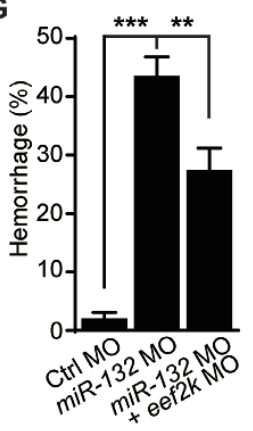

$\mathbf{L}$
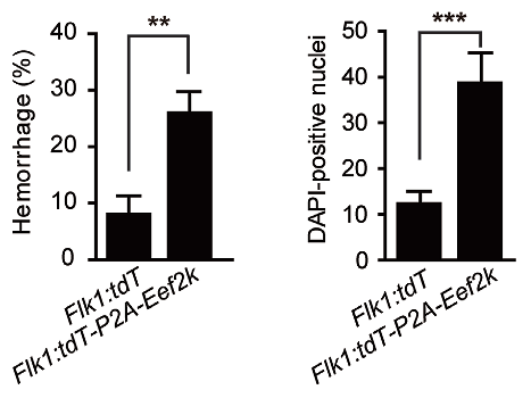

$\mathrm{H}$

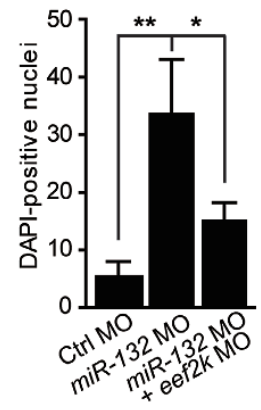

M

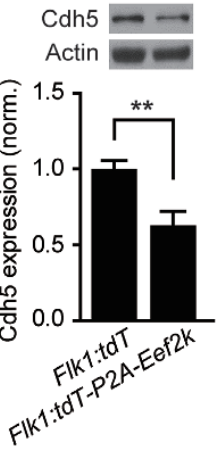

Figure 6 MiR-132 regulates Cdh5 expression and brain vascular integrity by directly targeting endothelial eef2k. (A) Schematic showing two potential miR-132 binding sites (in green) in the eef2k 3' UTR. Two mutants ("Mut-1", "Mut-2") of the eef2k 3' UTR were introduced by replacing the wildtype binding sequence (GACTGTT) with a mutant sequence (AGTCTCC, in red) at the two corresponding binding sites. The seed sequence of miR-132 is in blue. (B) Suppression of the luciferase activity of the eef2k 3' UTR reporter in HEK293 cells by miR-132. MiR-132 sensor served as a positive control. The experiments were repeated three times. (C) Requirement of the binding site 2 in the eef2k $3^{\prime}$ UTR for miR-132 effect. The experiments were repeated three times. (D, E) Effects of miR-132 overexpression (D) or knockdown (E) on eEF2K expression in zebrafish larvae. The experiments were repeated three times. (F) Pharmacological evidence showing eEF2K mediates the intracranial hemorrhage effect of miR-132 knockdown. NH125, eEF2K inhibitor; rapamycin, eEF2K non-specific activator. The experiments were repeated 5-8 times, and for each time, $>15$ embryos were examined for each group. (G-I) Rescue effect of eef2k knockdown on miR-132 knockdown-induced intracranial hemorrhage (G), DAPI leakage in the brain (H) and Cdh5 expression reduction (I) in zebrafish larvae. For $\mathbf{G}$, the experiments were repeated 6-7 times, and for each time, $>53$ embryos were examined for each group. For $\mathrm{H}, 8,8$, and 11 embryos were analyzed for "Ctrl MO", "miR-132 MO" and "miR-132 MO + eef2k $M O$ ", respectively. For I, the experiments were repeated four times. (J) Effect of neuron-specific miR-132 downregulation on endothelial eEF2K expression in zebrafish larvae. ECs were sorted by flow cytometry from 3-dpf $\mathrm{Tg}(\mathrm{Flk}$ 1:eGFP) larvae, which were injected with HuC:tdT or HuC:tdT-miR-132-S plasmid. The experiments were repeated three times. (K-M) Effects of EC-specific Eef2k overexpression on intracranial hemorrhage (K), DAPI leakage in the brain (L), and Cdh5 expression (M) of zebrafish larvae. The Eef2k was cloned into Flk1:tdT-P2A vector ("Flk1:tdT-P2A-Eef2k"). Expression of tdT in ECs ("Flk1:t$d T^{\prime \prime}$ ) served as a control. For $\mathbf{K}$, the experiments were repeated nine times, and for each time, $>5$ embryos were examined for each group. For L, 12 and 13 embryos were analyzed for "Flk1:tdT" and "Flk1:tdT-P2A-Eef2k", respectively. For M, the experiments were repeated six times. Error bars, SEM. n.s., no significant; ${ }^{*} P<0.05,{ }^{* *} P<0.01,{ }^{* * *} P<0.001$ (unpaired twotailed Student's $t$-test for B, D, E, J-M; one-way ANOVA with post hoc Tukey's multiple comparison test for $\mathbf{C}$ and $\mathbf{I}$; one-way ANOVA with post hoc Bonferroni's multiple comparison test for $\mathbf{F}, \mathbf{G}$ and $\mathbf{H}$ ). 


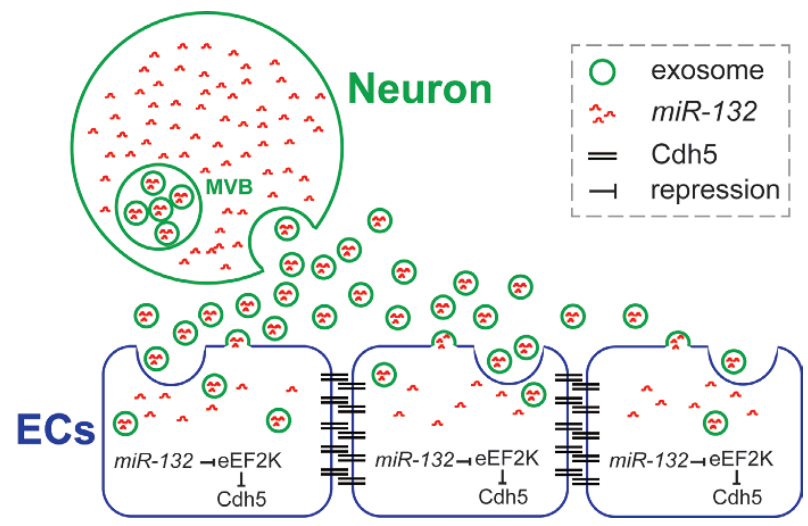

Figure 7 Working model. Neurons secrete miR-132-containing exosomes, which are internalized into brain ECs. In ECs, miR-132 upregulates Cdh5 expression via directly repressing eEF2K expression, promoting the development of brain vascular integrity.

nance of brain vascular integrity in adult brain.

\section{Exosomes are a novel avenue for neurovascular commu- nication}

Increasing evidence indicates that exosome-mediated intercellular communication plays important roles in both physiological and pathological processes in neural systems $[16,17]$. Under physiological conditions, neurons can transfer $m i R-124 a$ in exosomes to astrocytes to regulate the expression of astroglial glutamate transporters, which is important for the maintenance of extracellular glutamate levels [47]. Synapsin 1-containing exosomes secreted by astrocytes can be taken up by neurons and promote neurite outgrowth and neuronal survival [48]. Exosomes released by Schwann cells and oligodendrocytes can be taken up by neuronal axons to increase neurite growth, enhance axonal regeneration and improve neuronal survival $[49,50]$. Under pathological conditions, exosomes have been found to increase the propagation of neurotoxic proteins in neurodegenerative diseases [51-53], and aggravate brain tumor metastasis and progression [14]. During neural development, cultured embryonic rat cortical neurons can release exosomes [54]. In vivo studies in Drosophila larvae showed that motor neurons can secrete Wingless-associated and Synaptotagmin 4-containing exosomes to promote the synaptic development and plasticity $[11,18]$. In the present study, we showed that neuronal exosomes can also be internalized by brain ECs to mediate the neural regulation of brain vascular development. As release of neuronal exosomes can be upregulated by increased neural activity $[32,54]$, neural activity may regulate the brain vascular development and permeability through exosome release. As the contents of exosomes are abundant, other factors besides $m i R-132$ in neuronal exosomes may also participate in the neural regulation of brain vascular integrity. It will be of great future interest to examine the roles of other agents present in neuronal exosomes in neurovascular crosstalk.

The exosome is a suitable vehicle for drug delivery because of its high biocompatibility and low immunogenicity [55]. In addition, formulated nanoparticle-mediated miR-132 delivery into ECs can improve EC survival in vitro and EC transplantation efficiency in mice [56]. Therefore, application of miR-132-containing exosomes may be a safe and efficient future strategy for alleviating brain vascular dysfunctions associated with neurological and brain vascular diseases.

Our findings open an exciting avenue for the study of neuronal exosome-mediated neurovascular interactions. Further elucidation of neuronal exosomal functions in the regulation of brain vasculature should be of important biological and clinical significance.

\section{Materials and Methods}

\section{Zebrafish husbandry}

Adult zebrafish (Dario rerio) were maintained with an automatic fish housing system (ESEN, China) at $28{ }^{\circ} \mathrm{C}$ following standard protocols. The AB/WT, $\operatorname{Tg}(\mathrm{Flk} 1$ :eGFP), $\operatorname{Tg}(\mathrm{Flk} 1: \mathrm{RFP})$, $\operatorname{Tg}($ Gatal:DsRed) and $\operatorname{Tg}(\mathrm{HuC}$ :GFP) zebrafish were described previously [57, 58]. Zebrafish embryos were raised under a $14 \mathrm{~h}-10$ h light-dark cycle in $10 \%$ Hanks' solution that consisted of 140 $\mathrm{mM} \mathrm{NaCl}, 5.4 \mathrm{mM} \mathrm{KCl}, 0.25 \mathrm{mM} \mathrm{Na}_{2} \mathrm{HPO}_{4}, 0.44 \mathrm{mM} \mathrm{KH}_{2} \mathrm{PO}_{4}$, $1.3 \mathrm{mM} \mathrm{CaCl}_{2}, 1.0 \mathrm{mM} \mathrm{MgSO}_{4}$, and $4.2 \mathrm{mM} \mathrm{NaHCO}_{3}(\mathrm{pH}=7.2)$. $0.003 \%$ 1-phenyl-2-thiourea (PTU) (Sigma, P7629) was added in the Hanks' solution to prevent pigment formation of zebrafish embryos. Zebrafish chorion was removed at $1 \mathrm{dpf}$ with the treatment of $2 \mathrm{mg} / \mathrm{ml}$ pronase (Calbiochem, 53702), which was diluted in the Hanks' solution. All animal use and handling procedures were approved by Institute of Neuroscience, Chinese Academy of Sciences.

\section{Morpholino oligonucleotides and microinjection}

Morpholino oligonucleotides (MOs) were purchased from Gene Tools (Philomath, OR). Lyophilized MOs were dissolved in nuclease-free water. Zebrafish embryos were microinjected at the onecell stage with $8 \mathrm{ng} m i R-132 \mathrm{MO}, 8 \mathrm{ng}$ miR-132 loop MO, $0.5 \mathrm{ng}$ Cdh 5 MO, 0.5 - 1 ng eef $2 k$ MO, $4 \mathrm{ng} n$ SMase $2 \mathrm{MO}$, or equivalent control MO. In miR-132 rescue experiments, $8 \mathrm{ng} m i R-132 \mathrm{MO}$ was co-injected with $20 \mathrm{nM}$ miR-132 RNA or control RNA (Genepharma). The sequences of MOs or RNAs used are as follows. miR-132 MO: 5'-AGCGACCATGGCTGTAGACTGTTAC-3' miR-132 loop MO: 5'-GGCTGTAGACTGTTACCAAAAATTC-3'

Cdh5 MO: 5'-TACAAGACCGTCTACCTTTCCAATC-3' nSMase2 MO: 5'-CCACCTGCACCTGCACAAAACAACA-3' eef $2 \mathrm{kMO}$ : 5'-AGCTCCTCTTCAGCCATGATGCCCC-3' control MO: 5'-CCTCTTACCTCAGTTACAATTTATA-3' 
miR-132 RNA: 5'-UAACAGUCUACAGCCAUGGUCG-3' control RNA: 5'-UUGUACUACACAAAAGUACUG-3'

\section{CRISPR/Cas9-mediated mutation of miR-132 in zebrafish embryos}

The CRISPR (clustered regularly interspaced short palindromic repeats)/Cas9 system was applied to introduce $m i R-132$ gene mutation in zebrafish embryos as previously reported [58-60]. The zebrafish codon-optimized $\operatorname{Cas} 9$ (zCas 9 ) expression plasmid pGH-T7-zCas 9 was linearized by $\mathrm{XbaI}$ and used as a template for zCas 9 mRNA synthesis in vitro with mMACHINE T7 Ultra kit (Ambion, AM1345). The sequence of $m i R-132$ guide RNA (gRNA) (5'-TTGGTAACAGTCTACAGCCA-3') was designed to target the sequence of mature miR-132 (Supplementary information, Figure $\mathrm{S} 4 \mathrm{~A}$ ). A pair of oligonucleotides containing the miR-132 gRNA sequence were annealed and cloned into the pT7-gRNA plasmid. Then miR-132 gRNA was synthesized with MAXIscript T7 kit (Ambion, AM1312M) and purified with mirVana miRNA isolation kit (Ambion, AM1560). 600 pg zCas9 mRNA and 100 pg miR-132 gRNA were co-injected into zebrafish embryos at one-cell stage. The miR-132 gene mutation in F0 embryos was examined by PCR and sequencing analysis with primers (forward: 5'-CGCCTCGAGCAGTCTACAGTCATGGCTACTGACG-3'; reverse: 5'-GCGTCTAGACCTGTTCACTTGCATGCAAGG-3'). The knockout efficiency of mature $m i R-132$ transcription was examined by real-time PCR, and hemorrhagic phenotypes were examined in F0 embryos which carried miR-132 mutations.

\section{Generation of pericyte reporter zebrafish line Ki(pdgfr $\beta$ :- Gal4)}

The Ki(pdgfr $\beta: G a 14)$ line was generated with CRISPR/ Cas9-mediated knockin method as previously reported [58]. Briefly, the $p d g f r \beta$ gRNA, which targets to the intron between the last and second last exons, was synthesized. For the donor plasmid, the left and right arms of $p d g f r \beta$ were amplified from the zebrafish genomic DNA. The left arm, gal4 fragment and right arm were ligated into the PM19-T vector. $1 \mathrm{nl}$ RNAase free liquid mixture, which contained 800 pg zCas 9 mRNA, 80-100 pg pdgfr $\beta$ gRNA and $15 \mathrm{pg} p d g f r \beta$ donor plasmid, was injected into zebrafish embryos at one-cell stage. The embryos were raised to adulthood for founder screening. To screen $\operatorname{Ki}(\mathrm{pdgfr} \beta: \mathrm{Gal} 4)$ founders, adult fish were crossed with AB/WT zebrafish, the genomic DNA was extracted at $1 \mathrm{dpf}$, and the germline transition was detected by PCR.

\section{In vivo confocal imaging}

Larvae at 3-dpf were embedded in 1\% low-melting agarose (Invitrogen, 16520-050) without anesthetic at room temperature. Imaging was carried out with an Olympus Fluoview 1000 confocal microscopy (Olympus, Japan). Lumplfl 40× (W/IR; NA, 0.80) and XLumplfl 20× (W/IR; NA, 0.95) objective lenses (Olympus) were used. The z-step of imaging ranged from 2 to $3 \mu \mathrm{m}$. To detect the internalization of CD63-GFP-labeled neuronal exosomes into brain endothelial cells (ECs) in intact larvae, a Lumplfl $40 \times(\mathrm{W} /$ IR; NA, 0.80) objective lens was used with a z-step of $0.5 \mu \mathrm{m}$. Time-lapse images were taken with a 15 -min interval. Raw images were processed with deconvolution (Huygens Essential) to reduce the convolution effect produced by microscope optics and then thresholding with the Triangle method (ImageJ).

\section{Detection of brain vascular integrity}

$10 \mathrm{nl}$ mixture of DAPI $(0.8 \mathrm{mg} / \mathrm{ml})$ and $10-\mathrm{kDa}$ Alex-568 dextran $(10 \mathrm{mg} / \mathrm{ml})$ was injected into the blood circulation system of 3-dpf zebrafish larvae through the common cardinal vein. After 30 min, images were taken for calculating the number of DAPI-positive parenchymal nuclei outside brain vessels as previously described [24]. To detect leakage sites, 100-nm red fluorescent beads were injected into the blood circulation system and time-lapse images were taken immediately after the injection.

\section{Transmission electron microscopy}

Larvae at $3 \mathrm{dpf}$ were fixed in $2 \%$ glutaraldehyde in $0.1 \mathrm{M}$ phosphate buffer $(\mathrm{pH}=7.4)$ overnight at $4{ }^{\circ} \mathrm{C}$. Preparations were then washed, post-fixed with $1 \%$ osmium tetraoxide, dehydrated and embedded. Sections (70 $\mathrm{nm}$ in thickness) were cut and observed by transmission electron microscopy (JEM-1230).

\section{Calculation of pericyte coverage of brain blood vessels}

The numbers of brain blood vessel segments and pericytes in the zebrafish brain were quantitatively analyzed by using Brain Angiotome 1.1 [57]. The pericyte coverage of brain blood vessels was defined as the average number of pericytes for each brain blood vessel segment.

\section{Flow cytometry}

To compare the expression level of miR-132 in zebrafish ECs and neurons, $\operatorname{Tg}(\mathrm{Flk} 1$ :eGFP) and $\operatorname{Tg}(\mathrm{HuC}$ :GFP) larvae were used for EC and neuron sorting, respectively. To examine the effect of neuronal miR-132 manipulations on the expression of $m i R-132$ and eEF2K in ECs, $\operatorname{Tg}(\mathrm{Flk} 1$ :eGFP) larvae microinjected with $H u C: t-$ dT, HuC:tdT-miR-132 or HuC:tdT-miR-132-S were used for both neuron and EC sorting. Zebrafish embryos were dechorionated with pronase at $1 \mathrm{dpf}$. Embryos at $3 \mathrm{dpf}$ were suspended with PBS containing 2\% Fetal Bovine Serum (FBS), and pipetted up and down gently to remove the yolk. Embryos were then digested with $0.25 \%$ trypsin at $28{ }^{\circ} \mathrm{C}$ for $30 \mathrm{~min}$. Dissociated cells were collected by centrifugation, suspended with PBS containing $2 \%$ FBS, and then filtered with $40-\mu \mathrm{m}$ cell strainer (BD Falcon, 352340). The cell suspension was used to sort out neurons or ECs by a MoFlo XDP flow cytometer (Beckman, ML99030) with 488-nm and 560nm lasers.

\section{Rat primary cortical neuron culture and transfection}

Primary cortical neurons were prepared from E 16 Sprague-Dawley rat embryos as previously reported [54]. In brief, cerebral cortices were dissected and digested with $0.125 \%$ trypsin at $37{ }^{\circ} \mathrm{C}$ for $10 \mathrm{~min}$. Dissociated cells were filtered with $70-\mu \mathrm{m}$ cell strainer (BD Falcon, 352360), collected by centrifugation and then plated onto Petri dishes coated with $0.01 \mathrm{mg} / \mathrm{ml}$ Poly-D-Lysine (Sigma, P6407). Neurons were maintained at $37{ }^{\circ} \mathrm{C}$ in a humidified atmosphere of $5 \% \mathrm{CO}_{2}$ and cultured in Neurobasal medium (Invitrogen, 21103049) supplemented with Glutamax (Invitrogen, 35050061), B27 (Invitrogen, 17504044) and 5\% FBS (Biochrom, S0115/S0615) for the first day. From the next day, neurons were cultured in serum-free medium and half of the medium was replaced every 3 days. To manipulate the expression of $m i R$ 132 or label exosomes in cultured neurons, dissociated rat cortical neurons were transfected with $2 \mu \mathrm{g}$ plasmid or $100 \mathrm{nM}$ FAMtagged miR-132 (Genepharma) with a Rat Neuron Nucleofector 
Kit (Amaxa, VPG1003) by using an electroporator (Amaxa, Program O-03). The transfected cells were plated onto Poly-D-Lysine coated 6-well plates and cultured as described above.

\section{Purification, identification, labeling and treatment of rat neuronal exosomes}

Neuronal exosomes were purified from the conditioned medium of rat cortical neurons cultured 5 or 9 days in vitro (DIV). All transfected neurons were cultured 5 DIV in culture plates and exosomes were isolated by Total Exosome Isolation Reagent (Invitrogen, 4478359). Untransfected neurons were cultured 9 DIV in petri dishes and exosomes were isolated by multi-step centrifugation as previously described [61]. The isolated pellets were suspended in $1 \times$ PBS for exosome characterization and culture experiments. For electron microscopy, $10 \mu \mathrm{l}$ suspended pellets were applied onto carbon-coated copper grids. After the sample was dry, micrographs were taken with a calibrated magnification of 100000 fold by transmission electron microscope (JEM-1230). For nanoparticle tracking analysis, suspended pellets were diluted in $1 \mathrm{ml}$ PBS and applied to NanoSight (NS 300) to automatically measure the average diameter and concentration. To examine the uptake of neuronal exosomes by ECs in vitro, neuronal exosomes were labeled using a PKH67 green fluorescent labeling kit (Sigma-Aldrich, MINI67) or isolated from rat cortical neurons transfected with Fugw:CD63GFP plasmid. The labeled neuronal exosomes were then incubated with the mouse brain microvascular EC line (b.End3) at $37^{\circ} \mathrm{C}$ for $12 \mathrm{~h}$. The exosome-treated b.End 3 cells were then fixed and imaged. To examine the level of endothelial $m i R-132$ after neuronal exosome treatment, b.End 3 cells were incubated with the isolated neuronal exosomes at $37{ }^{\circ} \mathrm{C}$ for $24 \mathrm{~h}$. The addition of exosomes isolated from $50 \mathrm{ml}$ neuronal conditioned media into $0.5 \mathrm{ml} \mathrm{ECs}$ culture medium was counted as concentrating 100 times $(100 \times)$.

\section{Plasmid constructions}

To increase the expression of $m i R-132$, zebrafish pri-miR-132 was amplified from the zebrafish genomic DNA by using primers (forward: 5'-CGCCTCGAGCAGTCTACAGTCATGGCTACTGACG-3'; reverse: 5'-GCGTCTAGACCTGTTCACTTGCATGCAAGG-3') and cloned into $H u C: t d T$, pCS2:GFP or Fugw vectors. To reduce $m i R-132$ expression, $m i R-132$ sponge ( $m i R$ $132-S$ ) containing 10 repeated $m i R-132$ antisense sequences was synthesized by GeneArt and cloned into $H u C: t d T, F l k 1: t d T$ or Fugw vectors. The Eef $2 k$ was amplified from mouse cDNA with primers (forward: 5'-CGCCTTAAGCAAGCTGGTGATGTTGAAGAAAATCCTGGTCCTATGGCAGACGAAGACCTCATCTTC-3'; reverse: 5'- CGCGGATCCCGCTCTAGATTATTCCTCCATCTGGGCCCA-3') and cloned into Flk1:tdT-P2A vector. To label neuronal exosomes in vivo and in vitro, human $C D 63$ GFP (System Biosciences, CYTO120-VA-1) was cloned into $\mathrm{HuC}$ or Fugw vectors. All clones were verified by sequencing analysis. $30 \mathrm{ng}$ plasmid was injected into zebrafish embryos at one-cell stage.

\section{Whole-mount in situ hybridization}

Zebrafish whole-mount in situ hybridization was performed as previously described $[22,62]$ with a digoxigenin-labeled $m i R$ 132 Locked Nucleic Acid (LNA) probe (5'-CGACCATGGCTGTAGACTGTTA -3', Exiqon) and a scramble LNA probe (5'-GTGTAACACGTCTATACGCCCA-3', Exiqon). Embryos were incubated with corresponding probes (1:500) at $49{ }^{\circ} \mathrm{C}$ overnight. Digoxigenin was detected by anti-Digoxigenin AP-conjungated antibody (1:5 000, Roche 11093274910) and developed with NBT/ BCIP solution (Roche, 11681451001). Images were taken with an upright metallurgical microscope (Zeiss).

\section{Immunofluorescence}

Zebrafish whole-mount immunofluorescence was performed as previously reported [63]. Briefly, 2-dpf embryos were fixed in 4\% paraformaldehyde at $4{ }^{\circ} \mathrm{C}$ overnight. Then embryos were washed $4 \times 5 \mathrm{~min}$ in PBST (PBS $+0.5 \%$ Triton X-100) and incubated in a blocking solution (PBST $+10 \%$ normal goat serum $+1 \%$ BSA) for $2 \mathrm{~h}$ at room temperature. Embryos were then incubated with a rabbit anti-Cdh5 primary antibody (1:500, from Dr M Affolter's $\mathrm{Lab}$ ) in the blocking solution at $4{ }^{\circ} \mathrm{C}$ overnight. Embryos were further washed $6 \times 30 \mathrm{~min}$ in PBST and then incubated with an Alexa-568 goat anti-rabbit IgG secondary antibody (1:1000, Invitrogen) in the blocking solution at $4{ }^{\circ} \mathrm{C}$ overnight. After washing 3-5 times in PBST, images were taken with an Olympus Fluoview 1000 confocal microscope (Olympus, Japan).

\section{Western blotting}

Zebrafish embryos, cultured rat cortical neurons and isolated exosomes were lysed in RIPA buffer (Beyotime, P0013D) containing Protease Inhibitor Cocktail (Merck, 539134). Protein samples for CD63 detection were extracted by using Triton X-100 lysis buffer $(20 \mathrm{mM}$ Tris $\cdot \mathrm{HCl}$ at $\mathrm{pH} 7.4,137 \mathrm{mM} \mathrm{NaCl}, 1 \%$ Triton X-100, 2 mM EDTA, and 10\% glycerol) containing Protease Inhibitor Cocktail [12]. Protein concentration was measured by a Bio-Rad Protein Assay Kit and equal amounts of proteins were separated by SDS-PAGE and transferred onto PVDF membranes. Membranes were incubated in a blocking solution (TBS $+0.2 \%$ Triton $\mathrm{X}-100+5 \% \mathrm{BSA}$ ) for $2 \mathrm{~h}$ at room temperature and then probed with primary antibodies (as indicated below) overnight at $4{ }^{\circ} \mathrm{C}$. After washing $3 \times 15 \mathrm{~min}$ in TBST (TBS $+0.2 \%$ Triton $\mathrm{X}-100)$, membranes were incubated with HRP-conjugated secondary antibodies (Jackson) for $1 \mathrm{~h}$ at room temperature, rewashed and signals detected with the ECL Western blotting substrate (Pierce). The primary antibodies were anti-Cdh5 (Cell Signaling, 2500 ), anti- $\alpha$-catenin (BD Pharmingen, 610193), anti- $\beta$-catenin (Abcam, ab6302), anti-ZO1 (Invitrogen, 40-2200), anti-Claudin-5 (Invitrogen, 187364), anti-Occludin (Invitrogen, 71-1500), anti-N-cadherin (Invitrogen, 33-3900), anti-eEF2K (Cell Signaling, 3692), anti-Actin (Abmart, M20010), anti-CD63 (BD Pharmingen, 551458), anti-Calnexin (Abcam, ab22595), and anti-Alix (Cell Signaling, 2171).

\section{In vitro luciferase and in vivo reporter assays}

Two repeated miR-132 antisense sequences were cloned into psiCHECK-2 vector as a $m i R-132$ sensor. Zebrafish eef $2 k$ 3' UTR (500 bp) containing miR-132 target sequences was amplified from the zebrafish cDNA with primers (forward: $5^{\prime}-\mathrm{CG}-$ CCTCGAGTTTACAGATTGATCAAAATGGTTTA-3'; reverse: 5'-CGCTCTAGACGCGCGGCCGCTCCAGCAAAAGTGATCACACA-3') and cloned into psiCHECK-2 or psiCHECK-2:tdTomato vector. The mutant eef $2 k$ 3' UTR with mis-matched miR-132 binding sites was amplified using mutant primers. For in vitro luciferase assay, $100 \mathrm{ng}$ luciferase reporter plasmid was co-transfected with 400 ng pCS2:GFP or pCS2:miR-132 plasmid into cultured 
HEK293 cells using Lipofectamine 2000 (Invitrogen, 11668027). Luciferase activity was detected with a Dual-Luciferase Reporter Assay System (Promega, E1910) $24 \mathrm{~h}$ after transfection. For the in vivo reporter assay, linearized psiCHECK-2:tdTomato-eef $2 k$ 3' UTR and pCS2:GFP plasmids were used as the templates for tdTomato-eef $2 k$ 3'UTR and GFP mRNAs synthesis in vitro, respectively. The tdTomato-eef $2 k$ 3'UTR mRNA (50 pg) and GFP mRNA (30 pg), together with control RNA (200 pg) or miR-132 RNA (200 pg), were co-injected into zebrafish embryos at the onecell stage. Images were taken at $1 \mathrm{dpf}$ and the ratio of tdTomato to GFP fluorescence intensity was calculated with ImageJ.

\section{$R N A$ preparation and real-time $P C R$}

Total RNAs of zebrafish embryos, flow cytometry-sorted zebrafish neurons and ECs, cultured cells and isolated exosomes were extracted by using TRIzol reagent according to the manufacturer's instructions (Invitrogen, 15596018). The extracted total RNA was used to generate the first-strand cDNA by using PrimeScript reverse transcriptase (Takara, 2680A) with specific stem-loop primer for $m i R-132$ and random primer. The real-time PCR with SYBR Premix Ex Taq II (Takara) was performed on the cDNA to detect the relative $m i R-132$ expression. The relative RNA amount was calculated with the $\Delta \Delta \mathrm{Ct}$ method and normalized with U6 expression (as an internal control). The Taqman real-time PCR was only used to detect the absolute quantity of $m i R-132$ in different amount of neuronal exosomes. The primers used for real-time PCR are as follows.

miR-132 stem-loop primer:

5'-TGGAGCGACCGTGTCGTGGAGTCGGCTAATGGTCGCTCCACGACC-3'

miR-132 primers:

forward: 5'-GACACTCCAGCAGCGTAACAGTCTACAGC-

CATG-3'

reverse: 5'-ATAGAGCGGTGTCGTGGAGTCGGCTAATGGTC-3'

zebrafish U6 primers:

forward: 5'-ACTAAAATTGGAACGATACAGAGA-3'

reverse: 5'-AAAGATGGAACGCTTCACG-3'

mouse U6 primers:

forward: 5'-TCGCTTCGGCAGCACATA-3'

reverse: 5'-ACGAATTTGCGTGTCATCCT-3'

\section{Drug treatment}

1-Benzyl-3-cetyl-2-methylimidazolium iodide (NH125) (2.5 $\mu \mathrm{M}$, Tocris 3439), rapamycin (2 $\mu \mathrm{M}$, Merck 553211), spiroepoxide $(10 \mu \mathrm{M}$, Santa Cruz SC-202721) or equivalent DMSO was added into zebrafish culture medium at $1 \mathrm{dpf}$ after the chorion was removed. The medium was replaced with fresh medium containing corresponding drugs at $2 \mathrm{dpf}$ and phenotypes were characterized at 3 dpf.

\section{Statistical analysis}

The significance of difference between two groups was determined by using unpaired two-tailed Student's $t$-test. For multiple-group comparisons, one-way ANOVA was performed along with post hoc Bonferroni's or Tukey's multiple comparison test to assess statistical significance with a $95 \%$ confidence interval. Bonferroni's Multiple Comparison Test was used for groups with different experimental numbers, while Tukey's Multiple Comparison
Test was used for groups with same experimental numbers. Page's trend tests were applied for examining whether there is an increasing trend in the level of $m i R-132$ as the amount of exosomes increases in Figure 4D. Calculations were performed by using GraphPad Prism v5.0 software. Data were represented as mean \pm SEM in all figures except specifically indicated, and $P<0.05$ was considered to be statistically significant.

\section{Accession number}

The accession number for the microarray data is GEO: GSE85291 (http://www.ncbi.nlm.nih.gov/geo/).

\section{Acknowledgments}

We are grateful to Drs N Lawson for providing the $\mathrm{Tg}(\mathrm{Flk} 1$ :eGFP) line, B Zhang for providing the $\mathrm{Tg}(\mathrm{Flk} 1$ :RFP) and $\mathrm{Tg}(\mathrm{Ga}-$ ta1:DsRed) lines, M Affolter for providing the Cdh5 antibody for Immunofluorescence, Y Kong, LJ Pan and B Zhang for TEM analysis, Q Hu for image analysis, WF Jiang for flow cytometry, and J Yan for statistics. This work was supported by grants of the Key State Research Program of China (2012CB945101), National Natural Science Foundation of China (31325011, 31500849), China Wan-Ren Program, Shanghai Leading Talent Program, Shanghai Subject Chief Scientist Program (14XD1404100) and Yang-Fan Program (14YF1406600) from the Science and Technology Commission of Shanghai Municipality, and the Postdoctoral Research Foundation of China (2015T80458).

\section{Author Contributions}

JLD and BX conceived the project. BX, YZ and JLD designed the experiments. BX and $\mathrm{YZ}$ carried out the experiments and analyzed the data. XFD helped find the preliminary phenomenon. JL, HXZ, YY and JWB contributed to the generation of knockout and knockin zebrafish lines. HH contributed to TEM-relevant experiments. JLD, BX and YZ wrote the manuscript.

\section{Competing Financial Interests}

The authors declare no competing financial interests.

\section{References}

1 Abbott NJ, Ronnback L, Hansson E. Astrocyte-endothelial interactions at the blood-brain barrier. Nat Rev Neurosci 2006; 7:41-53.

2 Zlokovic BV. The blood-brain barrier in health and chronic neurodegenerative disorders. Neuron 2008; 57:178-201.

3 Tam SJ, Watts RJ. Connecting vascular and nervous system development: angiogenesis and the blood-brain barrier. Annu Rev Neurosci 2010; 33:379-408.

4 Obermeier B, Daneman R, Ransohoff RM. Development, maintenance and disruption of the blood-brain barrier. Nat Med 2013; 19:1584-1596.

5 Zhao Z, Nelson AR, Betsholtz C, Zlokovic BV. Establishment and dysfunction of the blood-brain barrier. Cell 2015; 163:1064-1078.

6 Stenman JM, Rajagopal J, Carroll TJ, Ishibashi M, McMahon J, McMahon AP. Canonical Wnt signaling regulates organ-specific assembly and differentiation of CNS vasculature. 
Science 2008; 322:1247-1250.

7 Daneman R, Agalliu D, Zhou L, Kuhnert F, Kuo C, Barres BA. Wnt/beta-catenin signaling is required for CNS, but not non-CNS, angiogenesis. Proc Natl Acad Sci USA 2009; 106:641-646.

8 Liebner S, Corada M, Bangsow T, et al. Wnt/beta-catenin signaling controls development of the blood-brain barrier. $J$ Cell Biol 2008; 183:409-417.

9 Alvarez Jl, Dodelet-Devillers A, Kebir H, et al. The Hedgehog pathway promotes blood-brain barrier integrity and CNS immune quiescence. Science 2011; 334:1727-1731.

10 Colombo M, Raposo G, Thery C. Biogenesis, secretion, and intercellular interactions of exosomes and other extracellular vesicles. Annu Rev Cell Dev Biol 2014; 30:255-289.

11 Korkut C, Li Y, Koles K, et al. Regulation of postsynaptic retrograde signaling by presynaptic exosome release. Neuron 2013; 77:1039-1046.

12 Kucharzewska P, Christianson HC, Welch JE, et al. Exosomes reflect the hypoxic status of glioma cells and mediate hypoxia-dependent activation of vascular cells during tumor development. Proc Natl Acad Sci USA 2013; 110:7312-7317.

13 Valadi H, Ekstrom K, Bossios A, Sjostrand M, Lee JJ, Lotvall JO. Exosome-mediated transfer of mRNAs and microRNAs is a novel mechanism of genetic exchange between cells. Nat Cell Biol 2007; 9:654-659.

14 Zhang L, Zhang S, Yao J, et al. Microenvironment-induced PTEN loss by exosomal microRNA primes brain metastasis outgrowth. Nature 2015; 527:100-104.

15 Kourembanas S. Exosomes: vehicles of intercellular signaling, biomarkers, and vectors of cell therapy. Annu Rev Physiol 2015; 77:13-27.

16 Rajendran L, Bali J, Barr MM, et al. Emerging roles of extracellular vesicles in the nervous system. J Neurosci 2014; 34:15482-15489.

17 Budnik V, Ruiz-Canada C, Wendler F. Extracellular vesicles round off communication in the nervous system. Nat Rev Neurosci 2016; 17:160-172.

18 Korkut C, Ataman B, Ramachandran P, et al. Trans-synaptic transmission of vesicular Wnt signals through Evi/Wntless. Cell 2009; 139:393-404.

19 Edbauer D, Neilson JR, Foster KA, et al. Regulation of synaptic structure and function by FMRP-associated microRNAs miR-125b and miR-132. Neuron 2010; 65:373-384.

20 Magill ST, Cambronne XA, Luikart BW, et al. microRNA-132 regulates dendritic growth and arborization of newborn neurons in the adult hippocampus. Proc Natl Acad Sci USA 2010; 107:20382-20387.

21 Ha M, Kim VN. Regulation of microRNA biogenesis. Nat Rev Mol Cell Biol 2014; 15:509-524.

22 Wienholds E, Kloosterman WP, Miska E, et al. MicroRNA expression in zebrafish embryonic development. Science 2005; 309:310-311.

23 Salta E, Lau P, Sala Frigerio C, et al. A self-organizing miR$132 / \mathrm{Ctbp} 2$ circuit regulates bimodal notch signals and glial progenitor fate choice during spinal cord maturation. Dev Cell 2014; 30:423-436.

24 Tam SJ, Richmond DL, Kaminker JS, et al. Death receptors DR6 and TROY regulate brain vascular development. Dev Cell 2012; 22:403-417.
25 Anand S, Majeti BK, Acevedo LM, et al. MicroRNA-132-mediated loss of p120RasGAP activates the endothelium to facilitate pathological angiogenesis. Nat Med 2010; 16:909-914.

26 Giannotta M, Trani M, Dejana E. VE-cadherin and endothelial adherens junctions: active guardians of vascular integrity. Dev Cell 2013; 26:441-454.

27 Montero-Balaguer M, Swirsding K, Orsenigo F, Cotelli F, Mione M, Dejana E. Stable vascular connections and remodeling require full expression of VE-cadherin in zebrafish embryos. PLOS ONE 2009; 4:e5772.

28 Siekmann AF, Standley C, Fogarty KE, Wolfe SA, Lawson ND. Chemokine signaling guides regional patterning of the first embryonic artery. Genes Dev 2009; 23:2272-2277.

29 Armulik A, Genove G, Mae M, et al. Pericytes regulate the blood-brain barrier. Nature 2010; 468:557-561.

30 Daneman R, Zhou L, Kebede AA, Barres BA. Pericytes are required for blood-brain barrier integrity during embryogenesis. Nature 2010; 468:562-566.

31 Ebert MS, Neilson JR, Sharp PA. MicroRNA sponges: competitive inhibitors of small RNAs in mammalian cells. Nat Methods 2007; 4:721-726.

32 Chivet M, Javalet C, Laulagnier K, Blot B, Hemming FJ, Sadoul R. Exosomes secreted by cortical neurons upon glutamatergic synapse activation specifically interact with neurons. J Extracell Vesicles 2014; 3:24722.

33 Trajkovic K, Hsu C, Chiantia S, et al. Ceramide triggers budding of exosome vesicles into multivesicular endosomes. Science 2008; 309:1244-1247.

34 Pigott CR, Mikolajek H, Moore CE, et al. Insights into the regulation of eukaryotic elongation factor 2 kinase and the interplay between its domains. Biochem J 2012; 442:105-118.

35 Tavares CD, Ferguson SB, Giles DH, et al. The molecular mechanism of eukaryotic elongation factor 2 kinase activation. J Biol Chem 2014; 289:23901-23916.

36 Hancock ML, Preitner N, Quan J, Flanagan JG. MicroRNA-132 is enriched in developing axons, locally regulates Rasa1 mRNA, and promotes axon extension. J Neurosci 2014: 34:66-78.

37 Marler KJ, Suetterlin P, Dopplapudi A, et al. BDNF promotes axon branching of retinal ganglion cells via miRNA-132 and p250GAP. J Neurosci 2014; 34:969-979.

38 Wayman GA, Davare M, Ando H, et al. An activity-regulated microRNA controls dendritic plasticity by down-regulating p250GAP. Proc Natl Acad Sci USA 2008; 105:9093-9098.

39 Mellios N, Sugihara H, Castro J, et al. miR-132, an experience-dependent microRNA, is essential for visual cortex plasticity. Nat Neurosci 2011; 14:1240-1242.

40 Tognini P, Putignano E, Coatti A, Pizzorusso T. Experience-dependent expression of miR-132 regulates ocular dominance plasticity. Nat Neurosci 2011; 14:1237-1239.

41 Argaw AT, Asp L, Zhang J, et al. Astrocyte-derived VEGF-A drives blood-brain barrier disruption in CNS inflammatory disease. J Clin Invest 2012; 122:2454-2468.

42 Matsuoka RL, Marass M, Avdesh A, et al. Radial glia regulate vascular patterning around the developing spinal cord. Elife 2016; 5:e20253.

43 Katare R, Riu F, Mitchell K, et al. Transplantation of human pericyte progenitor cells improves the repair of infarcted heart through activation of an angiogenic program involving mi- 
cro-RNA-132. Circ Res 2011; 109:894-906.

44 Kong H, Yin F, He F, et al. The Effect of miR-132, miR-146a, and miR-155 on MRP8/TLR4-induced astrocyte-related inflammation. J Mol Neurosci 2015; 57:28-37.

45 Prat A, Biernacki K, Wosik K, Antel JP. Glial cell influence on the human blood-brain barrier. Glia 2001; 36:145-155.

46 Saharinen P, Eklund L, Miettinen J, et al. Angiopoietins assemble distinct Tie2 signalling complexes in endothelial cellcell and cell-matrix contacts. Nat Cell Biol 2008; 10:527-537.

47 Morel L, Regan M, Higashimori H, et al. Neuronal exosomal miRNA-dependent translational regulation of astroglial glutamate transporter GLT1. J Biol Chem 2013; 288:7105-7116.

48 Wang S, Cesca F, Loers G, et al. Synapsin I is an oligomannose-carrying glycoprotein, acts as an oligomannose-binding lectin, and promotes neurite outgrowth and neuronal survival when released via glia-derived exosomes. J Neurosci 2011; 31:7275-7290.

49 Fruhbeis C, Frohlich D, Kuo WP, et al. Neurotransmitter-triggered transfer of exosomes mediates oligodendrocyte-neuron communication. PLoS Biol 2013; 11:e1001604.

50 Lopez-Verrilli MA, Picou F, Court FA. Schwann cell-derived exosomes enhance axonal regeneration in the peripheral nervous system. Glia 2013; 61:1795-1806.

51 Aguzzi A, Rajendran L. The transcellular spread of cytosolic amyloids, prions, and prionoids. Neuron 2009; 64:783-790.

52 Emmanouilidou E, Melachroinou K, Roumeliotis T, et al. Cell-produced alpha-synuclein is secreted in a calcium-dependent manner by exosomes and impacts neuronal survival. J Neurosci 2010; 30:6838-6851.

53 Bellingham SA, Guo BB, Coleman BM, Hill AF. Exosomes: vehicles for the transfer of toxic proteins associated with neurodegenerative diseases? Front Physiol 2012; 3:124.

54 Faure J, Lachenal G, Court M, et al. Exosomes are released by cultured cortical neurones. Mol Cell Neurosci 2006; 31:642-648.

55 EL Andaloussi S, Mager I, Breakefield XO, Wood MJ. Extracellular vesicles: biology and emerging therapeutic opportunities. Nat Rev Drug Discov 2013; 12:347-357.

56 Devalliere J, Chang WG, Andrejecsk JW, et al. Sustained delivery of proangiogenic microRNA-132 by nanoparticle transfection improves endothelial cell transplantation. FASEB J 2014; 28:908-922.

57 Chen Q, Jiang L, Li C, et al. Haemodynamics-driven developmental pruning of brain vasculature in zebrafish. PLoS Biol 2012; 10:e1001374

58 Li J, Zhang BB, Ren YG, et al. Intron targeting-mediated and endogenous gene integrity-maintaining knockin in zebrafish using the CRISPR/Cas9 system. Cell Res 2015; 25:634-637.

59 Rossi A, Kontarakis Z, Gerri C, et al. Genetic compensation induced by deleterious mutations but not gene knockdowns. Nature 2015; 524:230-233.

60 Shah AN, Davey CF, Whitebirch AC, Miller AC, Moens CB. Rapid reverse genetic screening using CRISPR in zebrafish. Nat Methods 2015; 12:535-540.

61 Thery C, Clayton A, Amigorena S, Raposo G. Isolation and characterization of exosomes from cell culture supernatants and biological fluids. Curr Protoc Cell Biol 2006; 30:3.22.2123.22.29.

62 Yu PC, Gu SY, Bu JW, Du JL. TRPC1 is essential for in vivo angiogenesis in zebrafish. Circ Res 2010; 106:1221-1232.

63 Blum Y, Belting HG, Ellertsdottir E, Herwig L, Luders F, Affolter M. Complex cell rearrangements during intersegmental vessel sprouting and vessel fusion in the zebrafish embryo. Dev Biol 2008; 316:312-322.

(Supplementary information is linked to the online version of the paper on the Cell Research website.)

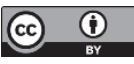

This work is licensed under a Creative Commons Attribution 4.0 Unported License. The images or other third party material in this article are included in the article's Creative Commons license, unless indicated otherwise in the credit line; if the material is not included under the Creative Commons license, users will need to obtain permission from the license holder to reproduce the material. To view a copy of this license, visit http:// creativecommons.org/licenses/by/4.0/

(C) The Author(s) 2017 